\title{
A Boltzmann-type kinetic model for misorientation distribution functions in two-dimensional fiber-texture polycrystalline grain growth
}

\author{
I. Yegorov ${ }^{\mathrm{a}, 1, *}$, C.E. Torres ${ }^{\mathrm{b}}$, M. Emelianenko ${ }^{\mathrm{a}}$ \\ ${ }^{a}$ George Mason University, Department of Mathematical Sciences, 4400 University Drive, MS: 3F2, Exploratory Hall, \\ Fairfax, Virginia, USA, 22030 \\ ${ }^{b}$ Departamento de Informatica and CCTVal - Centro Científico Tecnológico de Valparaíso, Universidad Técnica Federico Santa María, \\ Avenida España 1680, Valparaíso, Chile
}

\begin{abstract}
For mathematical modeling of polycrystalline materials, it is critical to understand how the statistics of evolving grain boundary networks depend on the set of laws that govern the dynamics at a microscopic scale. Such rules describe the motion of grain boundaries and their junctions, as well as criteria for the corresponding topological transitions. Although these laws have been known for quite some time, their precise role in the development of the networks' mesoscopic properties is not sufficiently clear. A possible way to establish a connection between statistical features of the whole networks and fundamental rules for the evolution of individual grain boundaries is to conduct numerical experiments via generally recognized large-scale simulation approaches. Alternatively, it is possible to develop less computationally expensive techniques such as kinetic models based on partial differential equations, while preserving essential characteristics of grain boundary networks. The aim of this paper is to develop a novel kinetic modeling approach for estimating number- and length-weighted misorientation distribution functions in the particular technologically important case of polycrystalline thin films with fiber textures and with anisotropic grain boundary energy densities. Our approach is tested by comparing the numerical results, which it gives for two specific benchmark examples, with the corresponding large-scale simulation results from the related literature. One auxiliary benchmark example is also considered.
\end{abstract}

Keywords: polycrystalline materials, grain growth, grain boundary, fiber texture, energy anisotropy, number-weighted misorientation distribution function, length-weighted misorientation distribution function, Boltzmann-type kinetic model.

\section{Introduction}

Polycrystalline materials such as metals and ceramics are comprised of single crystallites called grains. Interfaces between grains are called grain boundaries. Orientations, shapes, and arrangements of grains have a direct relationship to macroscopic properties of materials. For instance, the presence of grain boundaries decreases thermal and electrical conductivity affecting the performance of chips in microprocessors. Grain boundaries disrupt motion of dislocations through materials. Therefore, reducing crystallites' sizes is a common way to improve strength and fracture toughness of materials, as described by the well-known Hall-Petch relationship [1].

Grains' and grain boundaries' configurations, or microstructures, are determined by a variety of factors such as history of deformations, phase transitions, heat treatment, etc. In this paper, we are interested in the process of microstructural relaxation known as grain growth or coarsening. This phenomenon

\footnotetext{
${ }^{*}$ Corresponding author

Email addresses: ivanyegorov@gmail.com (I. Yegorov), ctorres@inf .utfsm.cl (C. E. Torres), memelian@gmu.edu (M. Emelianenko)

${ }^{1}$ Previous affiliation: Lomonosov Moscow State University, Department of Computational Mathematics and Cybernetics, Leninskie Gory, MSU, 2nd educational building, 119991, Moscow, Russia
}

refers to an increase in size of grains at a high temperature, occurring when recovery and recrystallization are complete, and the further reduction in the surface energy can be achieved only by decreasing the total area (in three-dimensional cases) or the total length (in two-dimensional cases) of grain boundaries.

For mathematical modeling of polycrystalline materials, it is critical to understand how the statistics of evolving grain boundary networks depend on the set of laws that govern the dynamics at a microscopic scale. Such rules describe the motion of grain boundaries and their junctions, as well as criteria for the corresponding topological transitions. Although these laws have been known for quite some time, their precise role in the development of the networks' mesoscopic properties is not sufficiently clear. A possible way to establish a connection between statistical features of the whole networks and fundamental rules for the evolution of individual grain boundaries is to conduct numerical experiments via generally recognized large-scale simulation approaches such as Monte Carlo techniques [2-8], phase field models [6,9-11], curvature-driven grain growth models [12-15], vertex models [16-18], diffusion generated motion models [19, 20], level set methods [21-23], etc.

Alternatively, it is possible to develop less computationally expensive techniques such as kinetic models based on partial differential equations, while preserving essential characteristics 
of grain boundary networks.

It is necessary to mention the Fradkov mean-field type kinetic model for two-dimensional grain growth [29-32] which is based on the classical von Neumann-Mullins law and considers time-evolving distribution functions for grain areas and topological classes (i. e., numbers of sides per grain). This approach is applied only to isotropic systems, because the von Neumann-Mullins relation and even the corresponding triple junction dragging generalization [33, § 4.6] require that the grain boundary energy density is constant. As opposed to the Fradkov model and its possible generalizations, our goal is to estimate so-called number- and length-weighted misorientation distribution functions (as considered in [4-8, 10, 17, 20, 22]) for anisotropic systems.

A novel framework for kinetic modeling of anisotropic microstructure evolution with accompanying topological transitions was proposed in [24] and applied to a simplified onedimensional model. A statistical mechanics perspective was adopted, and a system of grain boundaries was treated as a collection of interacting "particles", where the state of each particle was determined by parameters associated with the corresponding boundary. By exploiting grain boundaries' and particles' duality, two separate Boltzmann-type kinetic equations were derived, differing only in the number and choice of parameters constituting the corresponding state spaces.

The aim of this paper is to modify and extend the mentioned kinetic modeling approach to the two-dimensional case of polycrystalline thin films with so-called fiber textures [10]. In this case, grains have nearly identical orientations in the axial direction perpendicular to the considered film, but random radial orientations in the plane of the film [25]. Many technologically important characteristics of the films, such as their strength, conductivity, corrosion resistance or resistance against void formation, are correlated with fiber-texture characteristics of grain microstructures and grain boundary networks, in particular, with the misorientation distribution functions; see, for instance, [10, 26-28].

Note that the model developed in this paper is not exactly kinetic, because instead of the time variable, it operates with the varying total number of grain boundaries which is assumed to be decreasing in time.

We test our approach by comparing the numerical results, which it gives for two specific benchmark examples, with the corresponding large-scale simulation results presented in [10, 20]. One auxiliary benchmark example is also considered.

\section{Statement of the problem}

Consider a two-dimensional polycrystalline system with a perfect fiber texture $[3,5,10,20]$. This means the following:

- the grains and their boundaries have a common tilt axis (up to parallel transports) perpendicular to the plane of the simulation cell;

- there exists a single orientational degree of freedom for each of the grains, which is the azimuth (i.e., the angle of rotation) with respect to a fixed axis (up to parallel transports) in the plane of the simulation cell;

- during growth or shrinkage, the grains do not change their orientations.

In this context, the misorientation angle between two neighboring grains characterizes the corresponding boundary.

Suppose that scalar grain orientations and scalar misorientations between two neighboring grains have the smallest positive period $\alpha_{\max }$, and let us identify every two opposite misorientations with equal absolute values. Then we get the misorientations' symmetry with respect to $\frac{\alpha_{\max }}{2}$ on the interval $\left[0, \alpha_{\max }\right]$, and the sets of all orientations and misorientations up to the whole crystal symmetry can be taken as $\left[0, \alpha_{\max }\right)$ and $\left[0, \beta_{\max }\right]$, respectively, where

$$
\beta_{\max } \stackrel{\text { def }}{=} \frac{\alpha_{\max }}{2} .
$$

For instance, $\alpha_{\max }=\frac{\pi}{2}$ and $\beta_{\max }=\frac{\pi}{4}$ for the fourfold square symmetry, whereas $\alpha_{\max }=\frac{2 \pi}{3}$ and $\beta_{\max }=\frac{\pi}{3}$ for the regular hexagon symmetry.

Let

$$
\gamma:\left[0, \beta_{\max }\right] \rightarrow[0,+\infty)
$$

be the grain boundary energy density depending only on the misorientation variable.

Let $t \in[0, T]$ be the time variable, and let the total number and length of grain boundaries in the considered system be given by $N(t)$ and $L(t)$, respectively. Also denote the total number and length of boundaries with a particular misorientation $\beta \in\left[0, \beta_{\max }\right]$ by $N(\beta, t)$ and $L(\beta, t)$, respectively. Likewise, let $\bar{L}(t)$ be the average boundary length in the whole system, and let $\bar{L}(\beta, t)$ be the average length for a particular misorientation $\beta \in\left[0, \beta_{\max }\right]$ :

$$
\begin{array}{r}
\bar{L}(t) \stackrel{\text { def }}{=} \frac{L(t)}{N(t)}, \quad \bar{L}(\beta, t) \stackrel{\text { def }}{=} \frac{L(\beta, t)}{N(\beta, t)} \\
\forall \beta \in\left[0, \beta_{\max }\right] \quad \forall t \in[0, T] .
\end{array}
$$

Now introduce the number- and length-weighted misorientation distribution functions (MDFs) [8, 10]:

$$
\begin{aligned}
& \rho_{n}(\beta, t) \stackrel{\text { def }}{=} \frac{N(\beta, t)}{N(t)}, \quad \rho_{l}(\beta, t) \stackrel{\text { def }}{=} \frac{L(\beta, t)}{L(t)} \\
& \forall \beta \in\left[0, \beta_{\text {max }}\right] \quad \forall t \in[0, T] .
\end{aligned}
$$

Note that the length-weighted MDF $\rho_{l}$ is often referred grain boundary character distribution (GBCD); see, for instance, [13].

From (3),(4) we obtain

$$
\frac{\rho_{l}(\beta, t)}{\rho_{n}(\beta, t)}=\frac{\bar{L}(\beta, t)}{\bar{L}(t)} \quad \forall \beta \in\left[0, \beta_{\max }\right] \quad \forall t \in[0, T] .
$$

The right-hand side of this equality represents a dimensionless average boundary length for a particular misorientation $\beta \in\left[0, \beta_{\max }\right]$. 
We describe topological transitions accompanying twodimensional grain growth as either neighbor switchings or grain disappearances; see, for instance, [8, 16, 18]. Assume that initial microstructures do not contain two-sided grains and that only three-sided grains are allowed to disappear. Then, in particular, transformations of three-sided grains into two-sided grains by neighbor switchings are forbidden, and, therefore, two-sided grains cannot appear. This assumption is natural for vertex models [16-18]. Furthermore, the results of Monte Carlo and phase field simulations introduced in $[5,6]$ show that number fractions of two-sided grains are likely to be small even for sufficiently wide classes of highly anisotropic systems.

Moreover, for the sake of convenience, let us impose an idealizing and simplifying assumption. Suppose that the number of grain boundaries changes with time not discretely but continuously. In this case, $N(\beta, t)$ and $N(t)$ are considered to be real-valued functions. Apply the same assumption to the functions $N_{s}(t)$ and $N_{d}(t)$ representing total numbers of neighbor switchings and grain disappearances occuring up to a varying time moment $t$. It is obvious that $N_{s}(0)=N_{d}(0)=0$.

Since in our considerations, the misorientation arguments are continuous rather than discrete, then, more precisely, for every $t \in[0, T], N(\cdot, t)$ and $L(\cdot, t)$ should be interpreted as the measure densities such that the number and the total length of boundaries with misorientations from a Borel set $B \subseteq\left[0, \beta_{\max }\right]$ at time $t$ are the integrals $\int_{B} N(\beta, t) d \beta$ and $\int_{B} L(\beta, t) d \beta$, respectively. In particular,

$$
\begin{array}{r}
\int_{0}^{\beta_{\max }} N(\beta, t) d \beta=N(t), \int_{0}^{\beta_{\max }} L(\beta, t) d \beta=L(t) \\
\forall t \in[0, T] .
\end{array}
$$

Hence, by taking (4) into account, we conclude that $\rho_{n}(\cdot, t)$, $\rho_{l}(\cdot, t)$ are indeed probability densities for all $t \in[0, T]$ :

$$
\int_{0}^{\beta_{\max }} \rho_{n}(\beta, t) d \beta=\int_{0}^{\beta_{\max }} \rho_{l}(\beta, t) d \beta=1 \quad \forall t \in[0, T] .
$$

Our goal is to investigate the number- and length-weighted MDFs (4). Due to (5), the problem of predicting both of them can be reduced to the problem of predicting, for instance, $\rho_{n}$ and the right-hand side of (5); see also [8]. In what follows, we develop a novel kinetic model for obtaining the number-weighted MDF and estimate the quotient (5) by means of additional arguments.

\section{Kinetic equation for the number-weighted MDF}

We consider four types of possible critical events:

- creation of a boundary with a particular misorientation as a result of a neighbor switching;

- removal of a boundary with a particular misorientation as a result of a neighbor switching;
- removal of a zero-length boundary with a particular misorientation as a result of a grain disappearance;

- removal of a nonzero-length boundary with a particular misorientation as a result of a grain disappearance.

Adopt the following notations:

- $\left[0, \beta_{\max }\right] \ni \beta \longrightarrow P^{s,+}(\beta, t)$ is the probability density for misorientations of boundaries created as a result of neighbor switchings at the moment $t$;

- $\left[0, \beta_{\max }\right] \ni \beta \longrightarrow P^{s,-}(\beta, t)$ is the probability density for misorientations of boundaries removed with zero length as a result of neighbor switchings at the moment $t$;

- $\left[0, \beta_{\max }\right] \ni \beta \longrightarrow P_{1}^{d}(\beta, t)$ is the probability density for misorientations of boundaries removed with zero length as a result of grain disappearances at the moment $t$;

- $\left[0, \beta_{\max }\right] \ni \beta \longrightarrow P_{2}^{d}(\beta, t)$ is the probability density for misorientations of boundaries removed with nonzero length as a result of grain disappearances at the moment $t$.

In the next section, the introduced probability densities will be expressed in terms of the sought-for number-weighted MDF.

Also let $\lambda(t)$ be the ratio of the neighbor switchings' rate to the grain disappearances' rate at the moment $t$.

Now let us derive a kinetic equation for the number-weighted MDF. This equation is going to be a modification of the equation introduced in $\left[8\right.$, subsection 2.2]. Fix $\beta \in\left[0, \beta_{\max }\right]$, $t \in[0, T]$, and take a real number $\Delta t \in[-t, T-t] \backslash\{0\}$ with a small absolute value. We have

$$
\begin{aligned}
& N(\beta, t+\Delta t)-N(\beta, t)= \\
&=\left(P^{s,+}(\beta, t)-\right.\left.P^{s,-}(\beta, t)\right) \cdot\left(N_{s}(t+\Delta t)-N_{s}(t)\right)- \\
&-P_{1}^{d}(\beta, t) \cdot\left(N_{d}(t+\Delta t)-N_{d}(t)\right)- \\
&-P_{2}^{d}(\beta, t) \cdot 2\left(N_{d}(t+\Delta t)-N_{d}(t)\right) .
\end{aligned}
$$

Here we assume that when a three-sided grain disappears, only one of its sides is eliminated with zero length. Due to (4), the left-hand side of (8) can be represented as

$$
\begin{aligned}
& N(\beta, t+\Delta t)-N(\beta, t) \approx \frac{\partial N(\beta, t)}{\partial t} \cdot \Delta t= \\
& \quad=\frac{\partial}{\partial t}\left(N(t) \cdot \rho_{n}(\beta, t)\right) \cdot \Delta t= \\
& \quad=\left(\frac{\partial N(t)}{\partial t} \cdot \rho_{n}(\beta, t)+N(t) \cdot \frac{\partial \rho_{n}(\beta, t)}{\partial t}\right) \cdot \Delta t .
\end{aligned}
$$

Note that $\frac{\partial N(t)}{\partial t}, \frac{\partial \rho_{n}(\beta, t)}{\partial t}$ are considered to be right partial derivatives for $t=0$ and as left partial derivatives for $t=T$. Divide both sides of (8) by the difference

$$
N(t+\Delta t)-N(t) \approx \frac{\partial N(t)}{\partial t} \cdot \Delta t .
$$

Since only three-sided grains can disappear at once, then

$$
\frac{N_{d}(t+\Delta t)-N_{d}(t)}{N(t+\Delta t)-N(t)}=-\frac{1}{3},
$$


which implies

$$
\begin{aligned}
& \frac{N_{s}(t+\Delta t)-N_{s}(t)}{N(t+\Delta t)-N(t)}=\frac{N_{s}(t+\Delta t)-N_{s}(t)}{N_{d}(t+\Delta t)-N_{d}(t)} . \\
& \quad \cdot \frac{N_{d}(t+\Delta t)-N_{d}(t)}{N(t+\Delta t)-N(t)}=-\frac{N_{s}(t+\Delta t)-N_{s}(t)}{3 \cdot\left(N_{d}(t+\Delta t)-N_{d}(t)\right)} \approx \\
& \approx-\frac{\frac{\partial N_{s}(t)}{\partial t}}{3 \cdot \frac{\partial N_{d}(t)}{\partial t}}=-\frac{\lambda(t)}{3} .
\end{aligned}
$$

Finally, we get

$$
\begin{aligned}
\rho_{n}(\beta, t) & +N(t) \cdot \frac{\partial \rho_{n}(\beta, t)}{\partial N(t)}= \\
= & -\frac{\lambda(t)}{3} \cdot\left(P^{s,+}(\beta, t)-P^{s,-}(\beta, t)\right)+ \\
& +\frac{1}{3} \cdot P_{1}^{d}(\beta, t)+\frac{2}{3} \cdot P_{2}^{d}(\beta, t) .
\end{aligned}
$$

Since equation (9) does not contain any partial derivatives with respect to $t$, while $\lambda$ can be considered as a function of $N$, i.e., $\lambda=\lambda(N)$, and $P^{s,+}, P^{s,-}, P_{1}^{d}, P_{2}^{d}$ are supposed to be expressed in terms of $\rho_{n}$, then here it is possible to replace the dependence on $t$ with the dependence on $N$. This is reasonable also because of the fact that $N=N(t)$ is considered to be a strictly decreasing real-valued function; this implies the existence of the inverse function $t=t(N)$. For instance,

$$
\begin{aligned}
& \frac{d N(t)}{d t}=-g(N(t), t) \quad \forall t \in[0, T] \\
& g(0, t) \equiv 0 \text { and } g(\eta, t)>0 \quad \forall \eta>0 \quad \forall t \in[0, T] .
\end{aligned}
$$

Thus, we come to the equation

$$
\begin{aligned}
\rho_{n}(\beta, N) & +N \cdot \frac{\partial \rho_{n}(\beta, N)}{\partial N}= \\
= & -\frac{\lambda(N)}{3} \cdot\left(P^{s,+}(\beta, N)-P^{s,-}(\beta, N)\right)+ \\
& +\frac{1}{3} \cdot P_{1}^{d}(\beta, N)+\frac{2}{3} \cdot P_{2}^{d}(\beta, N) .
\end{aligned}
$$

Here

$$
\left.N_{0} \stackrel{\text { def }}{=} N\right|_{t=0},\left.\quad N_{\text {min }} \stackrel{\text { def }}{=} N\right|_{t=T}
$$

are parameters. The initial condition is taken as

$$
\rho_{n}\left(\beta, N_{0}\right)=\rho_{n}^{0}(\beta) \quad \forall \beta \in\left[0, \beta_{\max }\right],
$$

where $\rho_{n}^{0}$ is a specified initial number-weighted MDF.

If (10) holds, then this Cauchy problem has the following form in the variables $\beta, t$ :

$$
\left\{\begin{array}{c}
\rho_{n}(\beta, t)-\frac{N(t)}{g(N(t), t)} \cdot \frac{\partial \rho_{n}(\beta, t)}{\partial t}= \\
=-\frac{\lambda(t)}{3} \cdot\left(P^{s,+}(\beta, t)-P^{s,-}(\beta, t)\right)+ \\
+\frac{1}{3} \cdot P_{1}^{d}(\beta, t)+\frac{2}{3} \cdot P_{2}^{d}(\beta, t) \\
\forall \beta \in\left[0, \beta_{\max }\right] \quad \forall t \in[0, T], \\
\left.\rho_{n}(\beta, t)\right|_{t=0}=\rho_{n}^{0}(\beta) \quad \forall \beta \in\left[0, \beta_{\max }\right] .
\end{array}\right.
$$

Let us write a simple backward difference numerical scheme for Cauchy problem (11),(13):

$$
\begin{array}{r}
\rho_{n}(\beta, N)+N \cdot \frac{\rho_{n}(\beta, N-\Delta N)-\rho_{n}(\beta, N)}{-\Delta N}= \\
=-\frac{\lambda(N)}{3} \cdot\left(P^{s,+}(\beta, N)-P^{s,-}(\beta, N)\right)+ \\
\quad+\frac{1}{3} \cdot P_{1}^{d}(\beta, N)+\frac{2}{3} \cdot P_{2}^{d}(\beta, N),
\end{array}
$$

$$
\left\{\begin{aligned}
\rho_{n}(\beta, N-\Delta N)= & \rho_{n}(\beta, N)+\frac{\Delta N}{N} \cdot\left(\rho_{n}(\beta, N)+\right. \\
+ & \frac{\lambda(N)}{3} \cdot\left(P^{s,+}(\beta, N)-P^{s,-}(\beta, N)\right)- \\
& \left.-\frac{1}{3} \cdot P_{1}^{d}(\beta, N)-\frac{2}{3} \cdot P_{2}^{d}(\beta, N)\right),
\end{aligned}\right.
$$

$\Delta N>0$ is sufficiently small,

$\rho_{n}\left(\beta, N_{0}\right)=\rho_{n}^{0}(\beta)$.

\section{Representations of $P^{s,+}, P^{s,-}, P_{1}^{d}, P_{2}^{d}$}

We propose a modification and extension of the onedimensional approach introduced in [24] to our twodimensional case.

First, introduce the auxiliary function

$$
\begin{aligned}
& {\left[-2 \beta_{\max }, 2 \beta_{\max }\right] \ni \xi \longrightarrow} \\
& \longrightarrow \omega(\xi) \stackrel{\text { def }}{=} \begin{cases}|\xi|, & |\xi| \in\left[0, \beta_{\max }\right], \\
2 \beta_{\max }-|\xi|, & |\xi| \in\left(\beta_{\max }, 2 \beta_{\max }\right] .\end{cases}
\end{aligned}
$$

It transforms angles from $\left[-2 \beta_{\max }, 2 \beta_{\max }\right]$ to angles from $\left[0, \beta_{\max }\right]$ according to the symmetry with respect to zero and $\left(2 \beta_{\max }\right)$-periodicity of misorientations, which together imply, in particular, the symmetry with respect to $\beta_{\max }$ on $\left[0,2 \beta_{\max }\right]$.

According to Figures $1-4$, for all $\beta \in\left[0, \beta_{\max }\right]$ and $N \in$ $\left[N_{\text {min }}, N_{0}\right], P^{s,+}(\beta, N), P^{s,-}(\beta, N), P_{1}^{d}(\beta, N), P_{2}^{d}(\beta, N)$ can be represented in terms of the number-weighted MDF as

$$
\begin{aligned}
P^{s,+}(\beta, N) & =\frac{p^{s,+}(\beta, N)}{\int_{0}^{\max } p^{s,+}(\xi, N) d \xi}, \\
P^{s,-}(\beta, N) & =\frac{p^{s,-}(\beta, N)}{\int_{\max }^{\max } p^{s,-}(\xi, N) d \xi}, \\
P_{1}^{d}(\beta, N) & =\frac{\int_{0}^{\beta_{\max }} p_{1}^{d}(\xi, N) d \xi}{\beta_{2}^{d}(\beta, N)}, \\
P_{2}^{d}(\beta, N)= & \frac{p_{2}^{d}(\beta, N)}{\beta_{\max }} p_{2}^{d}(\xi, N) d \xi
\end{aligned}
$$


where

$$
\begin{aligned}
& p^{s,+}(\beta, N) \stackrel{\text { def }}{=} \\
& \stackrel{\text { def }}{=} \underset{\left(\beta_{1}, \beta_{2}\right) \in\left[-\beta_{\max }, \beta_{\max }\right] \times\left[-\beta_{\max }, \beta_{\max }\right]:}{ } \rho_{n}\left(\omega\left(\beta_{2}-\beta_{1}\right), N\right) . \\
& \gamma\left(\omega\left(\beta_{2}-\beta_{1}\right)\right) \geqslant \gamma(\beta) \\
& \cdot \rho_{n}\left(\left|\beta_{1}\right|, N\right) \cdot \rho_{n}\left(\left|\beta_{2}\right|, N\right) \cdot \rho_{n}\left(\omega\left(\beta+\beta_{1}\right), N\right) \cdot \\
& \text { - } \rho_{n}\left(\omega\left(\beta+\beta_{2}\right), N\right) d \beta_{1} d \beta_{2}, \\
& p^{s,-}(\beta, N) \stackrel{\text { def }}{=} \rho_{n}(\beta, N) . \\
& \iint_{\left(\beta_{1}, \beta_{2}\right) \in\left[-\beta_{\max }, \beta_{\max }\right] \times\left[-\beta_{\max }, \beta_{\max }\right]:} \rho_{n}\left(\left|\beta_{1}\right|, N\right) \cdot \\
& \gamma\left(\omega\left(\beta_{2}-\beta_{1}\right)\right) \leqslant \gamma(\beta) \\
& \cdot \rho_{n}\left(\left|\beta_{2}\right|, N\right) \cdot \rho_{n}\left(\omega\left(\beta+\beta_{1}\right), N\right) \cdot \\
& \text { - } \rho_{n}\left(\omega\left(\beta+\beta_{2}\right), N\right) d \beta_{1} d \beta_{2},
\end{aligned}
$$

$$
\begin{aligned}
& p_{1}^{d}(\beta, N) \stackrel{\text { def }}{=} \rho_{n}(\beta, N) \cdot \\
& \iint_{\substack{\left(\beta_{1}, \beta_{2}\right) \in\left[-\beta_{\max }, \beta_{\max }\right] \times\left[-\beta_{\max }, \beta_{\max }\right]: \\
\gamma\left(\omega\left(\beta_{2}-\beta_{1}\right)\right) \leqslant \min \left\{\gamma(\beta), \gamma\left(\left|\beta_{1}\right|\right)+\gamma\left(\beta_{2} \mid\right)\right\}}} \rho_{n}\left(\left|\beta_{1}\right|, N\right) \cdot \\
& \cdot \rho_{n}\left(\left|\beta_{2}\right|, N\right) \cdot \rho_{n}\left(\omega\left(\beta+\beta_{1}\right), N\right) \cdot \rho_{n}\left(\omega\left(\beta+\beta_{2}\right), N\right) . \\
& \rho_{n}\left(\omega\left(\beta_{2}-\beta_{1}\right), N\right) d \beta_{1} d \beta_{2},
\end{aligned}
$$$$
p_{2}^{d}(\beta, N) \stackrel{\text { def }}{=} \rho_{n}(\beta, N) .
$$

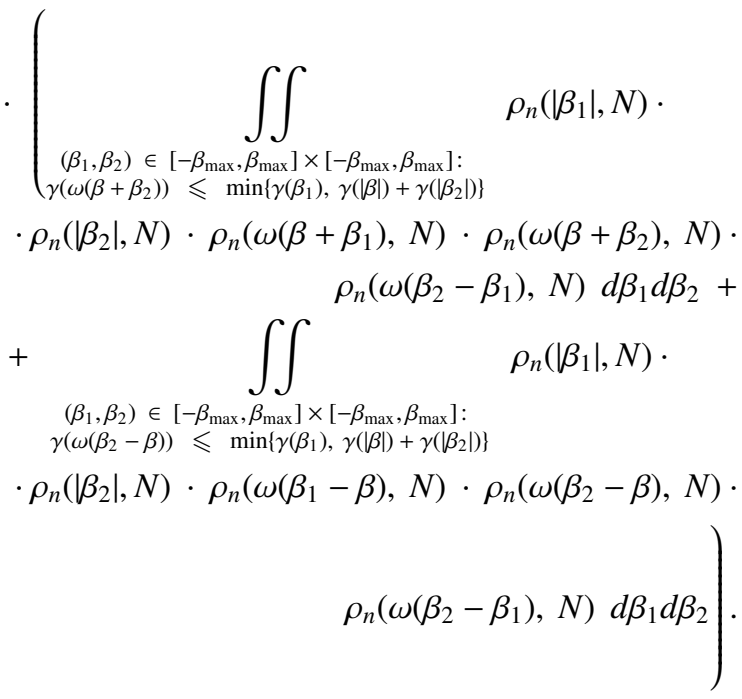

In (18)-(21), we determine the sets of integration by inequalities specifying the energy dissipation as a result of topological transitions. Note that we consider every grain disappearance as a combination of the following two consecutive events which should be energy dissipative:

- the neighbor switching which removes one zero-length boundary of a three-sided grain, generates a new zerolength boundary, and binds the remaining two boundaries of the destroyed grain into a two-sided grain;

- the instantaneous removal of the generated zero-length boundary together with the whole constructed two-sided grain.
Such interpretation is stipulated by the convention that after a grain disappearance, a new triple junction is located at the first collision point [18]. Besides, as in [24], we suppose the absence of correlations when writing the right-hand sides of (18)-(21). In order to be convinced that such assumption is reasonable and not too restrictive, we have to compare numerical results of our model with those obtained for the same initial data by some generally recognized large-scale simulation technique, for instance, by the phase field model described in [9-11]. This will be done in section 7 .

\section{Important remarks}

Remark 1. Consider the case when the initial numberweighted grain orientation distribution is uniform on the interval $\left[0, \alpha_{\max }\right]=\left[0,2 \beta_{\max }\right]$ (recall (1)). Let $\tilde{\rho}_{n}^{0}$ denote the probability density for the absolute value of the difference of two independent random variables equidistributed on $\left[0, \alpha_{\max }\right]$. Then

$$
\tilde{\rho}_{n}^{0}(\beta)= \begin{cases}\frac{2\left(\alpha_{\max }-\beta\right)}{\alpha_{\max }^{2}}, & \beta \in\left[0, \alpha_{\max }\right], \\ 0, & \beta \in \mathbb{R} \backslash\left[0, \alpha_{\max }\right] .\end{cases}
$$

By taking the misorientations' symmetry with respect to $\beta_{\max }$ on $\left[0, \alpha_{\max }\right]$ into account, we conclude that the initial numberweighted MDF $\left.\rho_{n}^{0} \stackrel{\text { def }}{=} \rho_{n}\right|_{N=N_{0}}$ is uniform on $\left[0, \beta_{\max }\right]$ :

$$
\begin{aligned}
& \rho_{n}^{0}(\beta)= \tilde{\rho}_{n}^{0}(\beta)+\tilde{\rho}_{n}^{0}\left(\alpha_{\max }-\beta\right)=\frac{1}{\beta_{\max }} \quad \forall \beta \in\left[0, \beta_{\max }\right] \\
& \rho_{n}^{0}(\beta)= \begin{cases}\frac{1}{\beta_{\max }}, & \beta \in\left[0, \beta_{\max }\right] \\
0, & \beta \in \mathbb{R} \backslash\left[0, \beta_{\max }\right]\end{cases}
\end{aligned}
$$

In addition to (22), it is natural to assume that the initial lengthweighted MDF $\left.\rho_{l}^{0} \stackrel{\text { def }}{=} \rho_{l}\right|_{N=N_{0}}$ is uniform on $\left[0, \beta_{\max }\right]$, i.e.,

$$
\rho_{l}^{0}(\beta)= \begin{cases}\frac{1}{\beta_{\max }}, & \beta \in\left[0, \beta_{\max }\right] \\ 0, & \beta \in \mathbb{R} \backslash\left[0, \beta_{\max }\right]\end{cases}
$$

Indeed, for the large-scale simulations within the framework of the papers $[10,20]$, the generated initial fiber textures fulfil (22),(23) with a fairly good degree of accuracy.

Remark 2. By the use of (18),(19), one can easily verify the equality

$$
\int_{0}^{\beta_{\max }} p^{s,+}(\beta, N) d \beta=\int_{0}^{\beta_{\max }} p^{s,-}(\beta, N) d \beta \quad \forall N \in\left[N_{\min }, N_{0}\right],
$$

which conforms with the fact that each neighbor switching removes a single boundary and creates one new boundary.

Remark 3. Now consider the first equality in numerical scheme (15) and suppose that the denominators in the righthand sides of (17) do not vanish. If at some point $(\beta, N) \in$ $\left[0, \beta_{\max }\right] \times\left[N_{\min }+\Delta N, N_{0}\right], \rho_{n}(\beta, N)=0$ and also $\rho_{n}(\xi, N) \geqslant 0$ 
for all $\xi \in\left[0, \beta_{\max }\right]$, then due to $(17)-(21), P^{s,+}(\beta, N) \geqslant 0$ and $P^{s,-}(\beta, N)=P_{1}^{d}(\beta, N)=P_{2}^{d}(\beta, N)=0$, which leads to $\rho_{n}(\beta, N-\Delta N) \geqslant 0$. Therefore, the solution to Cauchy problem (11),(13) should not take negative values if $\rho_{n}^{0}(\xi) \geqslant 0$ for all $\xi \in\left[0, \beta_{\max }\right]$ and $\int_{0}^{\beta_{\max }} \rho_{n}^{0}(\xi) d \xi>0$. Furthermore, by taking (15),(17) into account, one can easily verify that if $\int_{0}^{\beta_{\max }} \rho_{n}(\xi, N) d \xi=1$ then $\int_{0}^{\beta_{\max }} \rho_{n}(\xi, N-\Delta N) d \xi=1$.

Remark 4. In order to exclude negative values of an approximate solution to Cauchy problem (11),(13) which are possible in case of sufficiently large computational inaccuracies, we need to transform numerical scheme (15). The first equality in (15) can be written as

$$
\begin{array}{r}
\frac{\rho_{n}(\beta, N-\Delta N)-\rho_{n}(\beta, N)}{\Delta N}=A(\beta, N) \cdot \rho_{n}(\beta, N)+ \\
+B(\beta, N),
\end{array}
$$

where

$$
\begin{aligned}
A(\beta, N) \stackrel{\text { def }}{=} & \frac{1}{N} \cdot\left(1-\frac{\lambda(N)}{3} \cdot \frac{P^{s,-}(\beta, N)}{\rho_{n}(\beta, N)}-\right. \\
& \left.-\frac{1}{3} \cdot \frac{P_{1}^{d}(\beta, N)}{\rho_{n}(\beta, N)}-\frac{2}{3} \cdot \frac{P_{2}^{d}(\beta, N)}{\rho_{n}(\beta, N)}\right), \\
B(\beta, N) \stackrel{\text { def }}{=} & \frac{1}{N} \cdot \frac{\lambda(N)}{3} \cdot P^{s,+}(\beta, N) .
\end{aligned}
$$

In (26), the quotients $\frac{P^{s,-}(\beta, N)}{\rho_{n}(\beta, N)}, \frac{P_{1}^{d}(\beta, N)}{\rho_{n}(\beta, N)}, \frac{P_{2}^{d}(\beta, N)}{\rho_{n}(\beta, N)}$ are understood as the corresponding coefficients near $\rho_{n}(\beta, N)$ in (19)(21); such interpretation is valid, in particular, for $\rho_{n}(\beta, N)=0$. Consider (25) as an approximation of a linear one-dimensional ordinary differential equation with constant coefficients on the interval $[0, \Delta N]$ for a sufficiently small $\Delta N>0$ :

$$
\begin{aligned}
& \frac{\partial \rho_{n}(\beta, N+(-1) \cdot h)}{\partial h}=A(\beta, N) . \\
& \cdot \rho_{n}(\beta, N+(-1) \cdot h)+B(\beta, N), \quad h \in[0, \Delta N] .
\end{aligned}
$$

Then we can propose a numerical scheme based on a formula for the exact solution of (27):

$$
\begin{aligned}
& \rho_{n}(\beta, N-\Delta N)= \\
& =\left\{\begin{array}{l}
\rho_{n}(\beta, N) \cdot e^{A(\beta, N) \cdot \Delta N}+ \\
+\frac{B(\beta, N)}{A(\beta, N)} \cdot\left(e^{A(\beta, N) \cdot \Delta N}-1\right), \quad A(\beta, N) \neq 0,= \\
\rho_{n}(\beta, N)+B(\beta, N) \cdot \Delta N, \quad A(\beta, N)=0,
\end{array}\right. \\
& =\rho_{n}(\beta, N) \cdot e^{A(\beta, N) \cdot \Delta N}+ \\
& +B(\beta, N) \cdot\left(\Delta N+\sum_{k=2}^{\infty} \frac{(A(\beta, N))^{k-1} \cdot(\Delta N)^{k}}{k !}\right),
\end{aligned}
$$

$\Delta N>0$ is sufficiently small,

$$
\rho_{n}\left(\beta, N_{0}\right)=\rho_{n}^{0}(\beta) \text {. }
$$

For this scheme, the a priori order of accuracy is not less than for (15). Besides, an approximate solution obtained by (28) should not take negative values for nonnegative initial data, because in this case, $B(\beta, N) \geqslant 0$ due to (17),(18),(26), and

$$
\frac{1}{A(\beta, N)} \cdot\left(e^{A(\beta, N) \cdot \Delta N}-1\right)>0 \text { for } A(\beta, N) \in \mathbb{R} \backslash\{0\} .
$$

Remark 5. Due to (17)-(21), the probability densities $P^{s,+}, P^{s,-}, P_{1}^{d}, P_{2}^{d}$ explicitly depend not on the grain boundary energy density (2) itself but on the corresponding relation of order. However, the ratio $\lambda$ of the neighbor switchings' rate to the grain disappearances' rate is expected to depend directly on $\gamma$.

Note that in [34] $\lambda$ was obtained from large-scale simulations via a two-dimensional curvature-driven vertex model applied to an initially Monte Carlo generated microstructure. Moreover, a concise and informal discussion of possible ranges for $\lambda$ was given in [35]. Nevertheless, by the time of working on this paper, we had not seen any general approaches to estimate $\lambda$ described in the literature on mathematical modeling of grain growth. Let us propose our own heuristic method to estimate $\lambda$.

For every $N \in\left[N_{\min }, N_{0}\right]$, introduce the average grain boundary energy density and the fractions of low-energy and highenergy boundaries:

$$
\begin{aligned}
\bar{\gamma}(N) \stackrel{\text { def }}{=} \int_{0}^{\beta_{\max }} \gamma(\beta) \cdot \rho_{n}(\beta, N) d \beta, \\
\kappa_{\mathrm{le}}(N) \stackrel{\text { def }}{=} \int_{\beta \in\left[0, \beta_{\max }\right]: \gamma(\beta) \leqslant \bar{\gamma}(N)} \rho_{n}(\beta, N) d \beta, \\
\kappa_{\mathrm{he}}(N) \stackrel{\text { def }}{=} \int_{\beta \in\left[0, \beta_{\max }\right]: \gamma(\beta) \geqslant \bar{\gamma}(N)} \rho_{n}(\beta, N) d \beta \\
\forall N \in\left[N_{\min }, N_{0}\right] .
\end{aligned}
$$

Here grain boundaries with energy densities less or equal to the average value $\bar{\gamma}(N)$ are called low-energy, whereas grain boundaries with energy densities greater or equal to $\bar{\gamma}(N)$ are called high-energy. When $N=N_{0}$, i. e., $t=0$, according to (22), we have

$$
\begin{aligned}
& \bar{\gamma}_{0} \stackrel{\text { def }}{=} \bar{\gamma}\left(N_{0}\right)=\frac{1}{\beta_{\max }} \cdot \int_{0}^{\beta_{\max }} \gamma(\beta) d \beta, \\
& \kappa_{\mathrm{le}}\left(N_{0}\right)=\frac{1}{\beta_{\max }} \cdot \mu\left(\left\{\beta \in\left[0, \beta_{\max }\right]: \gamma(\beta) \leqslant \bar{\gamma}_{0}\right\}\right), \\
& \kappa_{\mathrm{he}}\left(N_{0}\right)=\frac{1}{\beta_{\max }} \cdot \mu\left(\left\{\beta \in\left[0, \beta_{\max }\right]: \gamma(\beta) \geqslant \bar{\gamma}_{0}\right\}\right),
\end{aligned}
$$

where $\mu$ denotes the Lebesgue measure on $\mathbb{R}$.

In the isotropic case when $\gamma(\beta) \equiv \bar{\gamma}_{0}$ for all $\beta \in\left[0, \beta_{\max }\right]$, we obtain that $\bar{\gamma}(N) \equiv \bar{\gamma}_{0}$ and $\kappa_{\mathrm{le}}(N) \equiv \kappa_{\mathrm{he}}(N) \equiv 1$ for every $N \in\left[N_{\min }, N_{0}\right]$. Suppose that in this isotropic case, the ratio $\lambda=\lambda(N)$ of the neighbor switchings' rate to the grain disappearances' rate is equal to some constant $\lambda_{\text {isotr }}>0$ for all $N \in\left[N_{\min }, N_{0}\right]$. For instance, in the computer simulations of A. P. Sprague, B. R. Patterson, and S. Grandhi [34] conducted for the isotropic case, the ratio of the cumulative number of 
neighbor switchings to the cumulative number of grain disappearances turned out to be nearly constant at 1.34 , which implies that $\lambda_{\text {isotr }} \approx 1.34$ for these simulations.

For anisotropic grain boundary energy densities, we estimate $\lambda$ as

$$
\begin{array}{r}
\lambda(N) \approx \lambda_{\text {isotr }} \cdot v\left(\frac{2 \cdot \kappa_{\mathrm{he}}(N)}{\kappa_{\mathrm{le}}(N)+\kappa_{\mathrm{he}}(N)}\right) \\
\forall N \in\left[N_{\mathrm{min}}, N_{0}\right],
\end{array}
$$

where $v:(0,2) \rightarrow(0,+\infty)$ is an increasing function such that $v(1)=1$; for instance, $v(x)=x$ for all $x \in(0,2)$. Here we adopt the assumption that $\lambda(N)$ and the quotient

$$
\frac{2 \cdot \kappa_{\mathrm{he}}(N)}{\kappa_{\mathrm{le}}(N)+\kappa_{\mathrm{he}}(N)}=\frac{\kappa_{\mathrm{he}}(N)}{\frac{1}{2}\left(\kappa_{\mathrm{le}}(N)+\kappa_{\mathrm{he}}(N)\right)}
$$

should increase or decrease together. This is based on the following natural heuristic hypotheses:

- a grain boundary removed as a result of a neighbor switching is more likely to be high-energy;

- a grain boundary removed with zero length as a result of a grain disappearance is more likely to be high-energy, while the boundary issuing from the corresponding opposite vertex of the shrinking three-sided grain and not belonging to the latter is more likely to be low-energy.

The condition $v(1)=1$ is stipulated by the fact that in the mentioned isotropic case, we have $\kappa_{\mathrm{le}}(N) \equiv \kappa_{\mathrm{he}}(N) \equiv$ $\frac{2 \cdot \kappa_{\mathrm{he}}(N)}{\kappa_{\mathrm{le}}(N)+\kappa_{\mathrm{he}}(N)} \equiv 1$ for every $N \in\left[N_{\min }, N_{0}\right]$.

Finally, note that for a fixed function $\gamma$ and for functions $\lambda$ taking values from a sufficiently wide realistic range (i. e., for not too small and not too large $\lambda$ ), the qualitative portraits of the number-weighted MDFs are not expected to differ from each other substantially, especially at final stages of texture evolutions, and the same for the length-weighted MDFs; the results of our numerical simulations conform with such expectations (see remark 1 in section 7). Indeed, the general energy reduction principles stimulating grain growth do not seem to be directly related to the quotient $\lambda$, although the rates of textures' tending to the resulting configurations may depend on $\lambda$. Besides, $\lambda$ is unlikely to grow or decrease infinitely during texture evolutions, because the greater/smaller the high-energy boundaries' fraction is, the greater/smaller the probabilities of their eliminations are expected to be (see (17), (19)-(21)).

\section{Representation of the length-weighted MDF}

In order to estimate the right-hand side of (5) and, consequently, the length-weighted $\mathrm{MDF} \rho_{l}$, let us apply a heuristic quantitative model which describes the mechanism of lowenergy boundaries' lengthening at triple junctions [4, 8, 36] simplistically. The phenomenon of low-energy boundaries' lengthening was justified experimentally [37].

As in [36], consider a two-dimensional geometrical stencil where one of the ends of a grain boundary with a scalar misorientation $\beta \in\left[0, \beta_{\max }\right]$ and, therefore, with the energy density $\gamma(\beta)$ meets two other boundaries having the average energy density $\bar{\gamma}(N)$. Note that in [4, 8] Read-Shockley type energy densities depending on the disorientation variable were considered, and for similar boundary lengthening models, $\bar{\gamma}(N)$ was replaced with the maximum possible value of the energy density. However, taking $\bar{\gamma}(N)$ here seems to be more accurate. To begin, the three boundaries are in an isotropic configuration, i. e., they have the same length equal to the average length $\bar{L}(N)$ and constitute three angles equal to $\frac{2 \pi}{3}=120^{\circ}$. Now we let the boundaries relax until the equilibrium triple line geometry is reached and calculate the resulting length of the first boundary having the energy density $\gamma(\beta)$. Then, under the assumption that

$$
\bar{\gamma}(N)>0 \quad \forall N \in\left[N_{\min }, N_{0}\right],
$$

we come to the following simplified estimate for the quotient (5):

$$
\begin{aligned}
& \frac{\bar{L}(\beta, N)}{\bar{L}(N)} \approx \\
& \approx\left\{\begin{array}{rr}
1+\frac{1}{2}\left(1-\frac{\sqrt{3}}{\tan \left(\arccos \left(\frac{\gamma(\beta)}{2 \bar{\gamma}(N)}\right)\right)}\right), & \frac{\gamma(\beta)}{\bar{\gamma}(N)} \leqslant \sqrt{3}, \\
0, & \frac{\gamma(\beta)}{\bar{\gamma}(N)}>\sqrt{3},
\end{array}\right. \\
& \forall N \in\left[N_{\text {min }}, N_{\text {interm }}\right] .
\end{aligned}
$$

The right-hand side of (33) tends to $\frac{3}{2}$ when $\gamma(\beta) \rightarrow+0$, equals 1 when $\gamma(\beta)=\bar{\gamma}(N)$, and vanishes if $\frac{\gamma(\beta)}{\bar{\gamma}(N)} \geqslant \sqrt{3}$. Here $N_{\text {interm }} \in\left(N_{\min }, N_{0}\right)$ is some constant; for instance, $N_{\text {interm }}=$ $\frac{N_{\min }+N_{0}}{2}$. Its presence is called forth by the fact that for anisotropic grain boundary energy densities and the uniform initial number- and length-weighted MDFs (22),(23), relation (33) is not expected to hold at initial stages of texture evolutions.

It is also necessary to mention that for highly anisotropic boundary energy densities, higher-order junctions such as quadruple junctions may become stable $[4,6,11,18,20]$. Our model does not explicitly operate with stable junctions of orders greater than 3 , although we can interpret a quadruple junction as two triple junctions connected through a common grain boundary with a relatively small length. Nevertheless, taking such short boundaries into account or neglecting them may substantially affect the number-weighted MDF but not the lengthweighted MDF.

\section{Numerical simulations}

Recall that we assume the uniformity of the initial numberand length-weighted MDFs according to (22),(23).

First, consider the isotropic case when $\gamma(\beta) \equiv$ const for every $\beta \in\left[0, \beta_{\max }\right]$. Then with the help of (11), (13), (17)-(22), one can easily verify that $\frac{\partial \rho_{n}(\beta, N)}{\partial N}=0$ for all $\beta \in\left[0, \beta_{\max }\right]$, $N \in\left[N_{\min }, N_{0}\right]$, and that, hence, the number-weighted MDF 
remains uniform during the whole observed process. Furthermore, in this case, the right-hand side of (33) equals 1 for all $\beta \in\left[0, \beta_{\max }\right], N \in\left[N_{\min }, N_{0}\right]$, and it is reasonable to choose $N_{\text {interm }}=N_{0}$, which leads to the uniformity of the lengthweighted MDF for every $N \in\left[N_{\min }, N_{0}\right]$. Thus, our model gives natural and trivial results under initial conditions (22),(23) if the boundary energy density is constant.

Now consider three nontrivial types of boundary energy densities (see Figure 5).

Type I. Read-Shockley boundary energy density $[2,4,7,10$, 20, 22]:

$$
\begin{aligned}
& {\left[0, \beta_{\max }\right] \ni \beta \longrightarrow \gamma_{\mathrm{I}}(\beta) \stackrel{\text { def }}{=}} \\
& \stackrel{\text { def }}{=} \begin{cases}\gamma_{l}, & \beta=0, \\
\gamma_{l}+\left(\gamma_{m}-\gamma_{l}\right) \cdot \frac{\beta}{\beta_{\mathrm{I}}} \cdot\left(1-\ln \left(\frac{\beta}{\beta_{\mathrm{I}}}\right)\right), & \beta \in\left(0, \beta_{\mathrm{I}}\right], \\
\gamma_{m}, & \beta \geqslant \beta_{\mathrm{I}},\end{cases}
\end{aligned}
$$

$\gamma_{l} \geqslant 0, \quad \gamma_{m}>\gamma_{l}, \quad \beta_{\mathrm{I}} \in\left(0, \beta_{\max }\right]$.

Type II. Modification of $\gamma_{\mathrm{I}}$ with an additional local minimum [10]:

$$
\begin{aligned}
& {\left[0, \beta_{\max }\right] \ni \beta \longrightarrow \gamma_{\mathrm{II}}(\beta) \stackrel{\text { def }}{=}} \\
& \stackrel{\text { def }}{=}\left\{\begin{array}{l}
\gamma_{\mathrm{I}}(\beta), \quad \beta \in\left[0, \beta_{\mathrm{II}}-\beta_{\mathrm{II}}^{\prime}\right], \\
\gamma_{l, 1}+\left(\gamma_{m}-\gamma_{l, 1}\right) \cdot \frac{\left|\beta-\beta_{\mathrm{II}}\right|}{\beta_{\mathrm{II}}^{\prime}} \cdot\left(1-\ln \left(\frac{\left|\beta-\beta_{\mathrm{II}}\right|}{\beta_{\mathrm{II}}^{\prime}}\right)\right), \\
\quad \beta \in\left[\beta_{\mathrm{II}}-\beta_{\mathrm{II}}^{\prime}, \beta_{\mathrm{II}}+\beta_{\mathrm{II}}^{\prime}\right] \backslash\left\{\beta_{\mathrm{II}}\right\}, \\
\gamma_{l, 1}, \quad \beta=\beta_{\mathrm{II}}, \\
\gamma_{m}, \quad \beta \geqslant \beta_{\mathrm{II}}+\beta_{\mathrm{II}}^{\prime},
\end{array}\right. \\
& \gamma_{l, 1} \in\left(\gamma_{l}, \gamma_{m}\right), \quad \beta_{\mathrm{II}} \in\left(\beta_{\mathrm{I}}, \beta_{\max }\right], \quad \beta_{\mathrm{II}}^{\prime} \in\left(0, \beta_{\mathrm{II}}-\beta_{\mathrm{I}}\right] .
\end{aligned}
$$

Type III. Concave parabolic boundary energy density [16]:

$$
\begin{aligned}
& {\left[0, \beta_{\max }\right] \ni \beta \longrightarrow \gamma_{\mathrm{III}}(\beta) \stackrel{\text { def }}{=}} \\
& \stackrel{\text { def }}{=} \gamma_{l, 2}+\left(\gamma_{m}-\gamma_{l, 2}\right) \cdot \frac{\beta \cdot\left(2 \beta_{\max }-\beta\right)}{\beta_{\max }^{2}} \text {, } \\
& \gamma_{l, 2} \in\left(0, \gamma_{m}\right) .
\end{aligned}
$$

As in [10], we take

$$
\begin{aligned}
& \alpha_{\text {max }}=\frac{\pi}{2}=90^{\circ}, \quad \beta_{\max }=\frac{\pi}{4}=45^{\circ}, \\
& \gamma_{l}=0, \quad \gamma_{m}=0.25, \quad \gamma_{l, 1}=0.1, \\
& \beta_{\mathrm{I}}=\frac{\pi}{12}=15^{\circ}, \quad \beta_{\mathrm{II}}=\frac{5 \pi}{24}=37.5^{\circ}, \quad \beta_{\mathrm{II}}^{\prime}=\frac{\pi}{18}=10^{\circ} .
\end{aligned}
$$

Besides, for $\gamma_{\mathrm{III}}$, we choose

$$
\gamma_{l, 2}=\frac{\gamma_{m}}{2}=0.125
$$

For each of the introduced boundary energy densities $\gamma_{\mathrm{I}}, \gamma_{\mathrm{II}}$, $\gamma_{\mathrm{III}}$, we consider the following two ways of determining the ratio $\lambda=\lambda(N)$ of the neighbor switchings' rate to the grain disappearances' rate (see remark 5 in section 5 and recall that the value 1.34 was obtained in [34]):
А) $\lambda(N) \equiv 1.34$

B) $\lambda$ is estimated according to (31) with $\lambda_{\text {isotr }}=1.34$ and $v(x) \equiv x$.

We take $N_{0}=300000$; this is reasonable if the initial number of grains is 100000 and the initial average number of sides per grain is 6 , because each grain boundary belongs to exactly two grains (see, for instance, [33, section 4.6.2]). In order to compute $\rho_{n}$, we apply numerical scheme (28) with $\Delta N=7500$ step-by-step till the very end at which $\left.\rho_{n}\right|_{N=0}$ is obtained by the use of $\left.\rho_{n}\right|_{N=\Delta N}$. When having an approximate $\rho_{n}$, we apply (33) with $N_{\text {interm }}=\frac{N_{0}}{2}=150000$ so as to estimate $\rho_{l}$ from $N=N_{\text {interm }}$ to $N=0$.

The results of our numerical simulations are presented in Figures 6-20. The following remarks can be made.

Remark 1. From Figures 6-9, 14-19, we see only a slight difference between cases $\mathbf{A}$ and $\mathbf{B}$, which conforms with our hypothesis at the end of remark 5 in section 5 .

Remark 2. Figures 6-9 show that:

- for the boundary energy density $\gamma_{\mathrm{I}}$ with a plateau stretching from $\beta=\beta_{\mathrm{I}}=\frac{\pi}{12}=15^{\circ}$ to $\beta=\beta_{\max }=\frac{\pi}{4}=45^{\circ}$, the number- and length-weighted MDFs are discontinuous at $\beta=\beta_{\mathrm{I}}$;

- for the boundary energy density $\gamma_{\mathrm{II}}$ with a plateau stretching from $\beta=\beta_{\mathrm{I}}$ to $\beta=\beta_{\mathrm{II}}-\beta_{\mathrm{II}}^{\prime}=\frac{11 \pi}{72}=27.5^{\circ}$, the number- and length-weighted MDFs are discontinuous at $\beta=\beta_{\mathrm{I}}$ and $\beta=\beta_{\mathrm{II}}-\beta_{\mathrm{II}}^{\prime}$;

- for the boundary energy density $\gamma_{\mathrm{III}}$ without any plateaus, the number- and length-weighted MDFs are continuous.

Indeed, for $\gamma_{\mathrm{I}}, \gamma_{\mathrm{II}}$, the probability densities $P^{s,-}(\cdot, N), P_{1}^{d}(\cdot, N)$, $P_{2}^{d}(\cdot, N)$ turn out to be discontinuous at the corresponding misorientations, which is not detected for $\gamma_{\mathrm{III}}$; see Figures 1013 for case $\mathbf{B}$, a similar situation is observed in case $\mathbf{A}$. This is related to the energy inequalities specifying the sets of integration in (19),(20). For misorientations $\beta$ near an energy plateau but not on it, these sets of integration are smaller than for plateau misorientations, where $P^{s,-}(\cdot, N)$ and $P_{1}^{d}(\cdot, N)$ therefore have wells at the beginning of the evolutions, i. e., for sufficiently large $N$. Subsequently, $P_{2}^{d}(\cdot, N)$ also becomes discontinuous, and the directions of the jumps of $P^{s,-}(\cdot, N)$ and $P_{1}^{d}(\cdot, N)$ change according to the directions of the jumps of $\rho_{n}(\cdot, N)$, since the right-hand sides of (19)-(21) are directly proportional to $\rho_{n}(\beta, N)$. Moreover, $\rho_{n}(\cdot, N)$ does not change the directions of its jumps.

These discontinuities are not seen explicitly on the histograms for the number- and length-weighted MDFs demonstrated in [10, Figures $8(a, b), 9(a, b)]$ and [20, Figure 3(b)] as applied to boundary energy densities of Types I,II (note that in [20] the Read-Shockley boundary energy density with the other parameters $\gamma_{l}=0.1$ and $\gamma_{m}=0.9$ was chosen). The possible deficiency of enabling such discontinuities is outweighed by the fact that our approach is rather general and relies on a smaller 
amount of properties and parameters than considered, for instance, by large-scale phase field models described in [6, 9-11]. In addition, this drawback does not seem to be crucial, because the detected discontinuities of the number- and length-weighted MDFs are sufficiently small and should not essentially violate the natural structures of the MDFs.

Besides, jumps of the number-weighted MDF with respect to the misorientation variable should not strongly interfere with using numerical scheme (28), since equation (11) contains a partial derivative only with respect to $N$. From Figures 6-20, some computational inaccuracies can be noticed closer to the end $N=0$, but they do not look significant.

Remark 3. From Figures 6-9, we observe a qualitative similarity with the simulation results presented in [10, section 3.3, Figures 8(a,b), 9(a,b)] and [20, Figure 3(b)] for boundary energy densities of Types I,II (Type III was not considered in $[10,20])$.

Recall that our model is not exactly kinetic, because it operates with the decreasing total number of grain boundaries instead of the time variable. This somewhat complicates the direct comparison. However, important common tendencies are clearly seen. For $\gamma_{\mathrm{I}}$ (similarly for $\left.\gamma_{\mathrm{III}}\right), \rho_{n}(\cdot, N)$ and $\rho_{l}(\cdot, N)$ get a single peak at $\beta=0$ corresponding to the unique local and simultaneously absolute minimum of this boundary energy density. For $\gamma_{\mathrm{II}}$, an extra peak occurs at $\beta=\beta_{\mathrm{II}}$, where $\gamma_{\mathrm{II}}$ has its second local minimum which is not absolute. For sufficiently large $N$, the left peak grows slightly faster than the right one. For small $N$, the left peak grows much faster than the right one.

Although the quantitative differences between our numerical results and what is shown in $[10$, section 3.3 , Figures 8(a,b), 9(a,b)], [20, Figure 3(b)] do not seem to be crucial, we do not obtain that for $\gamma_{\mathrm{II}}$ the final left peaks of the MDFs are substantially smaller than for $\gamma_{\mathrm{I}}$. Nevertheless, we do not know any physical reasons explaining why there should be such kind of distinction. Besides, in [10, section 3.3], it was noticed that the heights of the left and right peaks of the MDFs should be quite sensitive to the number of possible discrete misorientations considered by phase field models. In particular, the greater this number, the higher the left peaks are likely to be.

Also note that according to [10, section 3.1], N. Moelans, F. A. Spaepen, and P. Wollants did not observe large amounts of stable junctions of orders greater than 3 in their phase field simulations for boundary energy densities of Types I,II. Thus, the fact that our model operates with stable triple junctions but not with stable higher-order junctions does not seem to be very restrictive.

Remark 4. Figures 14,15 imply that relation (33) predicts a slightly smaller boundary lengthening at small misorientations than it follows from [10, section 3.3, Figures 8(a,b), 9(a,b)].

Remark 5. From Figures 6-9, we see that the number- and length-weighted MDFs tend to the limiting finite states when $N$ decreases. For small $N$, the MDFs evolve slightly and mainly at low misorientations. This conforms with the simulation results introduced in [10, section 3.3, Figures $8(a, b), 9(a, b)]$ and [20, Figure 3(b)], where approximate steady states of the MDFs were indicated.

Remark 6. Figures 16-19 show that when $N$ decreases, the average boundary energy density $\bar{\gamma}(N)$ and the fraction $\kappa_{\mathrm{he}}(N)$ of high-energy boundaries decrease, whereas the fraction $\kappa_{\mathrm{le}}(N)$ of low-energy boundaries increases. Such behaviour persists up to some slight computational inaccuracies observed for small $N$, and it is a priori evident due to the energy reducing nature of grain growth. Furthermore, these quantities eventually tend to the corresponding approximate steady states.

Remark 7. According to Figure 20 for case B, when $N$ decreases, the ratio $\lambda(N)$ of the neighbor switchings' rate to the grain disappearances' rate also decreases and eventually reaches its approximate steady state.

\section{Concluding remarks}

In this paper, we proposed a novel kinetic modeling approach for estimating number- and length-weighted misorientation distribution functions in the particular technologically important case of polycrystalline thin films with fiber textures and with anisotropic grain boundary energy densities. The numberweighted misorientation distribution function was obtained numerically by solving a Cauchy problem for the derived firstorder partial differential equation, in which the time variable was replaced with the varying total number of grain boundaries. The ratio of the length- and number-weighted misorientation distribution functions was estimated by the use of a specific boundary lengthening model.

Despite some possible shortcomings of the developed framework, it was successfully tested by comparing the numerical results, which it gave for two nontrivial benchmark examples, with the corresponding large-scale simulation results presented in $[10,20]$. One auxiliary benchmark example was also considered. At the same time, our kinetic modeling approach based on the derived partial differential equation is a priori less computationally expensive than the generally recognized large-scale simulation techniques mentioned in the introduction.

Our approach has both theoretical as well as practical value. It can be used to elucidate fundamental mechanisms of texture evolution in two-dimensional polycrystalline systems.

As a possible future work, we can consider an extension of the developed approach by taking nucleations of new grains into account. The current framework belongs to a pure-death category and does not allow for any sort of grain birth. This may account for some deviations from experiments, where socalled defects are allowed to nucleate at certain rates; see, for instance, [21]. To extend our model by including a nucleation term and therefore by reformulating the kinetic equation seems to be worth investigating. Here one of the main difficulties lies in identifying a suitable nucleation regime.

\section{Acknowledgements}

M. E. and I. Y. were supported in part by National Science Foundation grant DMS-1056821, C. E. T. was supported in part by Basal Project FB 0821. 


\section{References}

1. Smith, W., Hashemi, J. Foundations of Materials Science and Engineering. McGraw-Hill Education: London, 2009.

2. Grest, G. S., Srolovitz, D. J., Anderson, M. P. Computer simulation of grain growth - IV. Anisotropic grain boundary energies. Acta Metallurgica 1985; 33(3):509-520.

3. Saito, Y., Enomoto, M. Monte Carlo simulation of grain growth. ISIJ International 1992; 32(3):267-274.

4. Holm, E. A., Hassold, G. N., Miodownik, M. A. On misorientation distribution evolution during anisotropic grain growth. Acta Materialia 2001; 49:2981-2991.

5. Moldovan, D., Wolf, D., Phillpot, S. R., Haslam A. J. Mesoscopic simulation of two-dimensional grain growth with anisotropic grain-boundary properties. Philosophical Magazine A 2002; 82(7):1271-1297.

6. Upmanyu, M., Hassold, G. N., Kazaryan, A., Holm, E. A., Wang, Y., Patton, B., Srolovitz, D. J. Boundary mobility and energy anisotropy effects on microstructural evolution during grain growth. Interface Science 2002; 10(2):201-216.

7. Gruber, J., Miller, H. M., Hoffmann, T. D., Rohrer, G. S., Rollett, A. D. Misorientation texture development during grain growth. Part I: Simulation and experiment. Acta Materialia 2009; 57:6102-6112.

8. Gruber, J., Rohrer, G. S., Rollett, A. D. Misorientation texture development during grain growth. Part II: Theory. Acta Materialia 2010; 58:14-19.

9. Moelans, N., Blanpain, B., Wollants, P. Quantitative analysis of grain boundary properties in a generalized phase field model for grain growth in anisotropic systems. Physical Review B 2008; 78(2), 024113.

10. Moelans, N., Spaepen, F. A., Wollants P. Grain growth in thin films with a fiber texture studied by phase-field simulations and mean field modeling. Philosophical Magazine 2010; 90(1-4):501-523.

11. Chang, K., Moelans, N. Effect of grain boundary energy anisotropy on highly-textured grain structures studied by phase field simulations. Acta Materialia $2014 ;$ 64:443-454

12. Kinderlehrer, D., Lee, J., Livshits, I., Rollett, A., Ta'asan, S. Mesoscale simulation of grain growth. Materials Science Forum 2004; 467-470:10571062

13. Kinderlehrer, D., Livshits, I., Rohrer, G. S., Ta'asan, S., Yu, P. Mesoscale simulation of the evolution of the grain boundary character distribution. $\mathrm{Ma}$ terials Science Forum 2004; 467-470:1063-1068.

14. Kinderlehrer, D., Livshits, I., Ta'asan, S. A variational approach to modeling and simulation of grain growth. SIAM Journal on Scientific Computing 2006; 28(5):1694-1715.

15. Barmak, K., Eggeling, E., Emelianenko, M., Epshteyn, Y., Kinderlehrer, D., Sharp, R., Ta'asan, S. Materials microstructures: entropy and curvature-driven coarsening. Research Institute for Mathematical Sciences, Kyoto University 2014; 1881:71-91.

16. Henseler, R., Niethammer, B., Otto, F. A reduced model for simulating grain growth. Free Boundary Problems 2004; Volume 147 of the series ISNM International Series of Numerical Mathematics:177-187.

17. Barrales-Mora, L.A. 2D vertex modeling for the simulation of grain growth and related phenomena. Mathematics and Computers in Simulation 2010; 80:1411-1427.

18. Torres, C. E., Emelianenko, M., Golovaty, D., Kinderlehrer, D., Ta'asan, S Numerical analysis of the vertex models for simulating grain boundary networks. SIAM Journal on Applied Mathematics 2015; 75(2):762-786.

19. Elsey, M., Esedoglu, S., Smereka, P. Large scale simulation of normal grain growth via diffusion generated motion. Proceedings of the Royal Society A: Mathematical, Physical, and Engineering Sciences 2011; 467(2126):381401 .

20. Elsey, M., Esedoglu, S., Smereka, P. Simulations of anisotropic grain growth: Efficient algorithms and misorientation distributions. Acta Materialia 2013 ; 61(6):2033-2043.

21. Hallberg, H. A modified level set approach to 2D modeling of dynamic recrystallization. Modelling and Simulation in Materials Science and Engineering 2013; 21, 085012.

22. Hallberg, H. Influence of anisotropic grain boundary properties on the evolution of grain boundary character distribution during grain growth - A 2D level set study. Modelling and Simulation in Materials Science and Engineering 2014; 22(8), 085005.

23. Miessen, C., Liesenjohann, M., Barrales-Mora, L. A., Shvindlerman, L. S., Gottstein, G. An advanced level set approach to grain growth - Accounting for grain boundary anisotropy and finite triple junction mobility. Acta Materialia 2015 ; 99:39-48.

24. Barmak, K., Emelianenko, M., Golovaty, D., Kinderlehrer, D., Ta'asan, S. A new perspective on texture evolution. International Journal of Numerical Analysis and Modeling 2008; 5:93-108.

25. Thompson, C. V. Structure evolution during processing of polycrystalline films. Annual Review of Materials Science 2000; 30:159-190.

26. Vaidya, S., Sinha, A. K. Effect of texture and grain structure on electromigration in Al-0.5\%Cu thin films. Thin Solid Films 1981; 75(3):253-259.

27. Knorr, D. B., Rodbell, K. P. The role of texture in the electromigration behavior of pure aluminum lines. Journal of Applied Physics 1996; 79, 2409.

28. Gungor, A., Barmak, K., Rollett, A. D., Cabral, C., Harper, J. M. E. Texture and resistivity of dilute binary $\mathrm{Cu}(\mathrm{Al}), \mathrm{Cu}(\mathrm{In}), \mathrm{Cu}(\mathrm{Ti}), \mathrm{Cu}(\mathrm{Nb}), \mathrm{Cu}(\mathrm{Ir})$ and $\mathrm{Cu}(\mathrm{W})$ alloy thin films. Journal of Vacuum Science \& Technology B 2002; 20:2314-2319.

29. Fradkov, V. E. A theoretical investigation of two-dimensional grain growth in the "gas" approximation. Philosophical Magazine Letters 1988; 58:271275 .

30. Fradkov, V. E., Udler, D. G. 2D normal grain growth: Topological aspects. Advances in Physics 1994; 43:739-789.

31. Henseler, R., Herrmann, M., Niethammer, B., Velazquez, J. J. L. A kinetic model for grain growth. Kinetic and Related Models 2008; 1(4):591-617.

32. Herrmann, M., Laurencot, P., Niethammer, B. Self-similar solutions to a kinetic model for grain growth. Journal of Nonlinear Science 2012; 22(3):399-427

33. Gottstein, G., Shvindlerman, L. S. Grain Boundary Migration in Metals: Thermodynamics, Kinetics, Applications. Taylor \& Francis Group: Boca Raton, 2010.

34. Sprague, A. P., Patterson, B. R., Grandhi, S. Topological characteristics of two-dimensional grain growth - Simulation and analysis. Metallurgical and Materials Transactions A 2010; 41(3):592-602.

35. DeHoff, R. T. Metric and topological contributions to the rate of change of boundary length in two dimensional grain growth. Acta Materialia 1998; 46(14):5175-5185

36. Rohrer, G. S., Gruber, J., Rollett, A.D. A model for the origin of anisotropic grain boundary character distributions in polycrystalline materials. In Applications of Texture Analysis, Rollett, A. D. (Editor), Ceramic Transactions, 201. John Wiley \& Sons: Hoboken, New Jersey, 2009.

37. Dillon, S. J., Rohrer, G. S. Mechanism for the development of anisotropic grain boundary character distributions during normal grain growth. Acta $\mathrm{Ma}$ terialia 2009; 57(1):1-7. 
Fundamental laws governing 2D fiber-texture polyctrystalline grain growth with the corresponding topological transitions

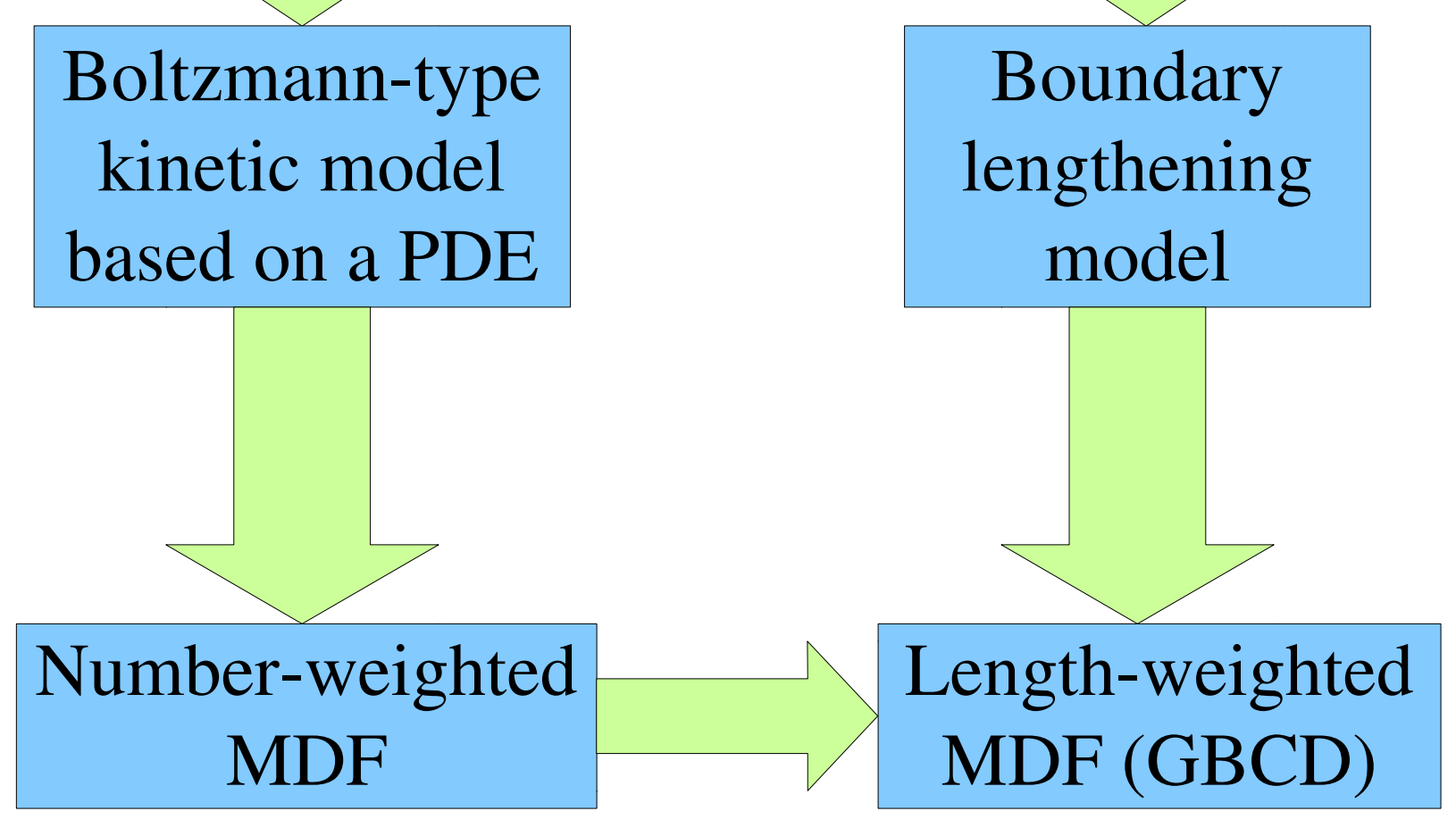

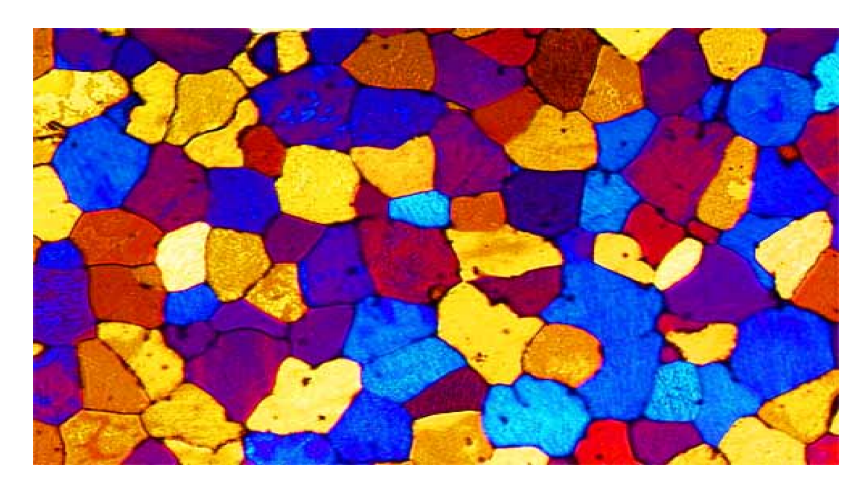
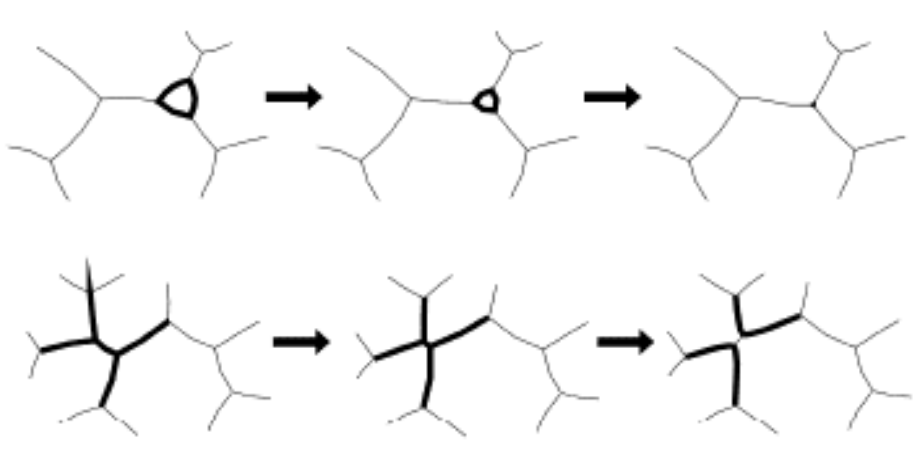

Despite some shortcomings of the developed framework, it is successfully tested by comparing the numerical results, which it gives for two important benchmark examples, with the corresponding large-scale simulation results obtained by phase field and diffusion generated motion models.

At the same time, it is is a priori less computationally expensive than large-scale direct simulation techniques. 


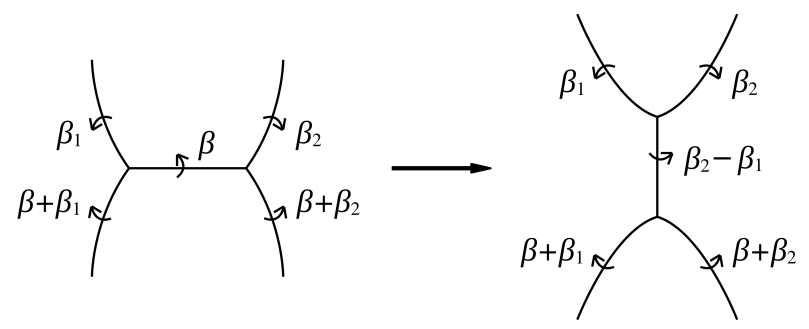

Figure 1: To formula (18). We focus on configurations before critical events.

1 


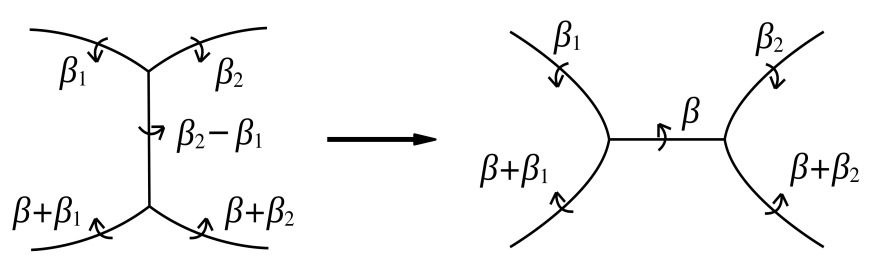

Figure 2: To formula (19). We focus on configurations before critical events. 

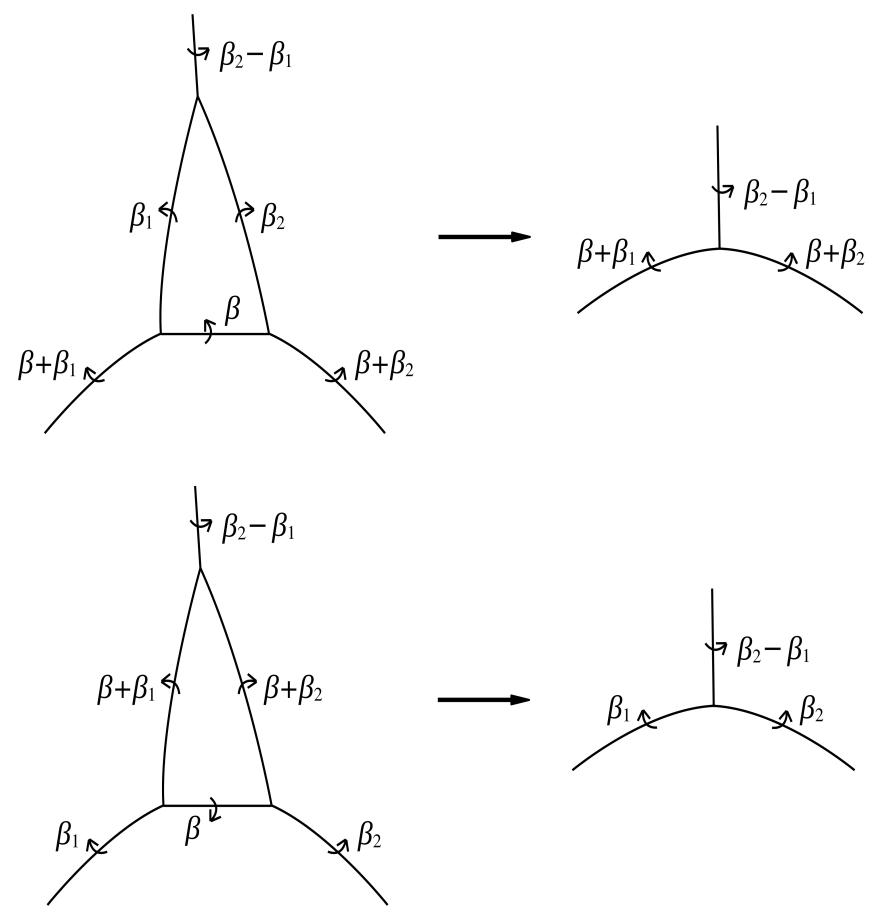

Figure 3: To formula (20). We focus on configurations before critical events. 

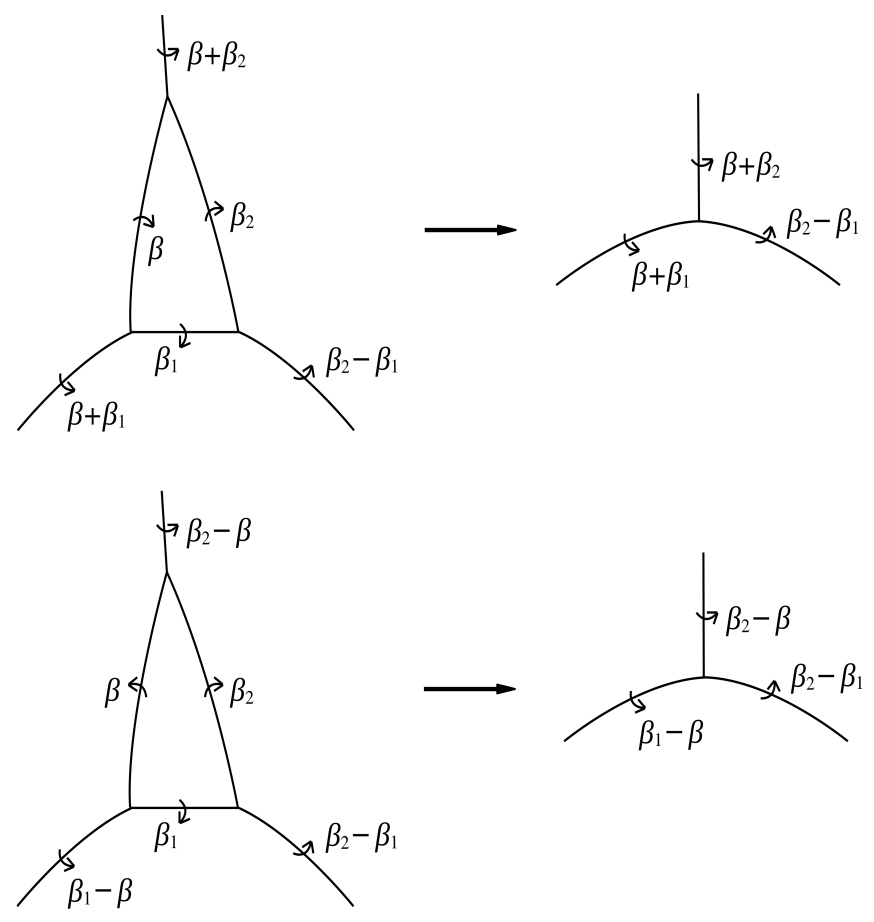

Figure 4: To formula (21). We focus on configurations before critical events. 

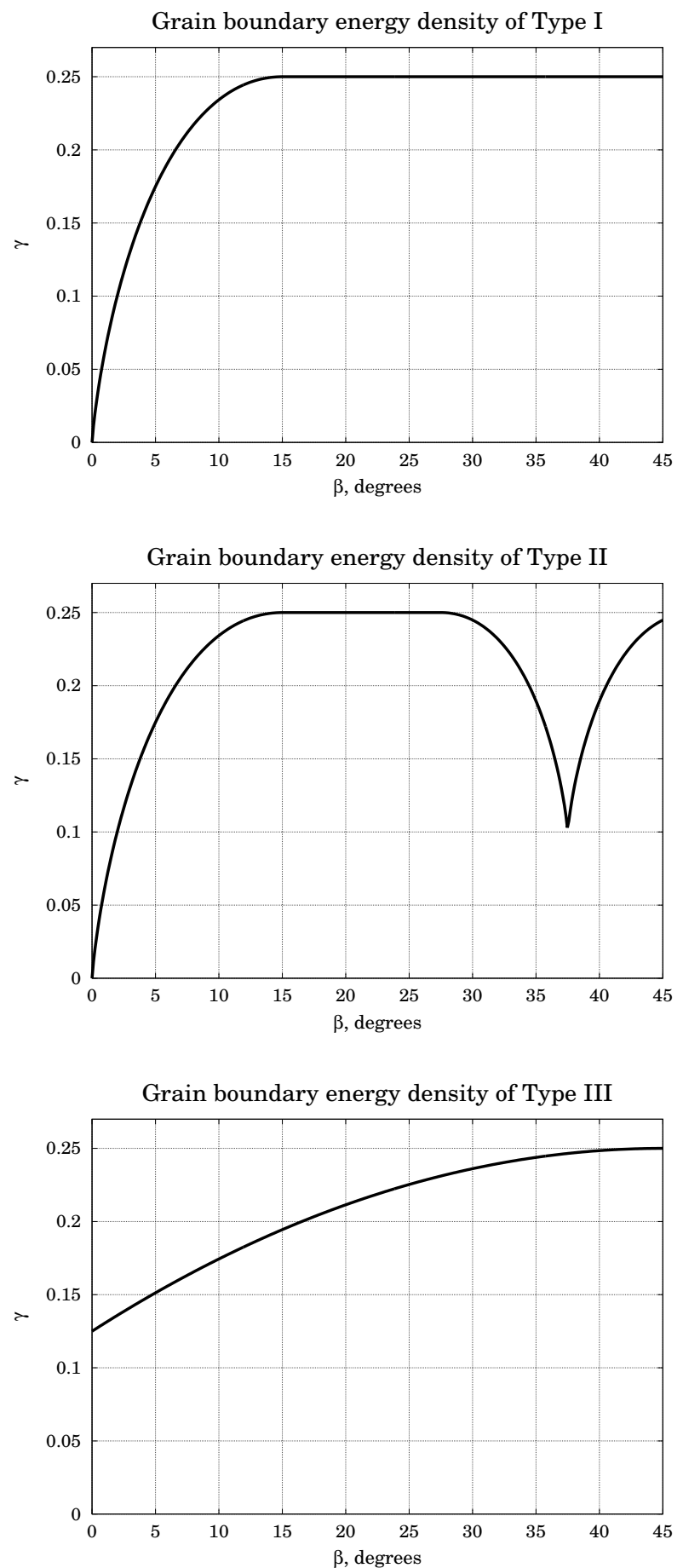

Figure 5: Three considered nontrivial types of boundary energy densities. 

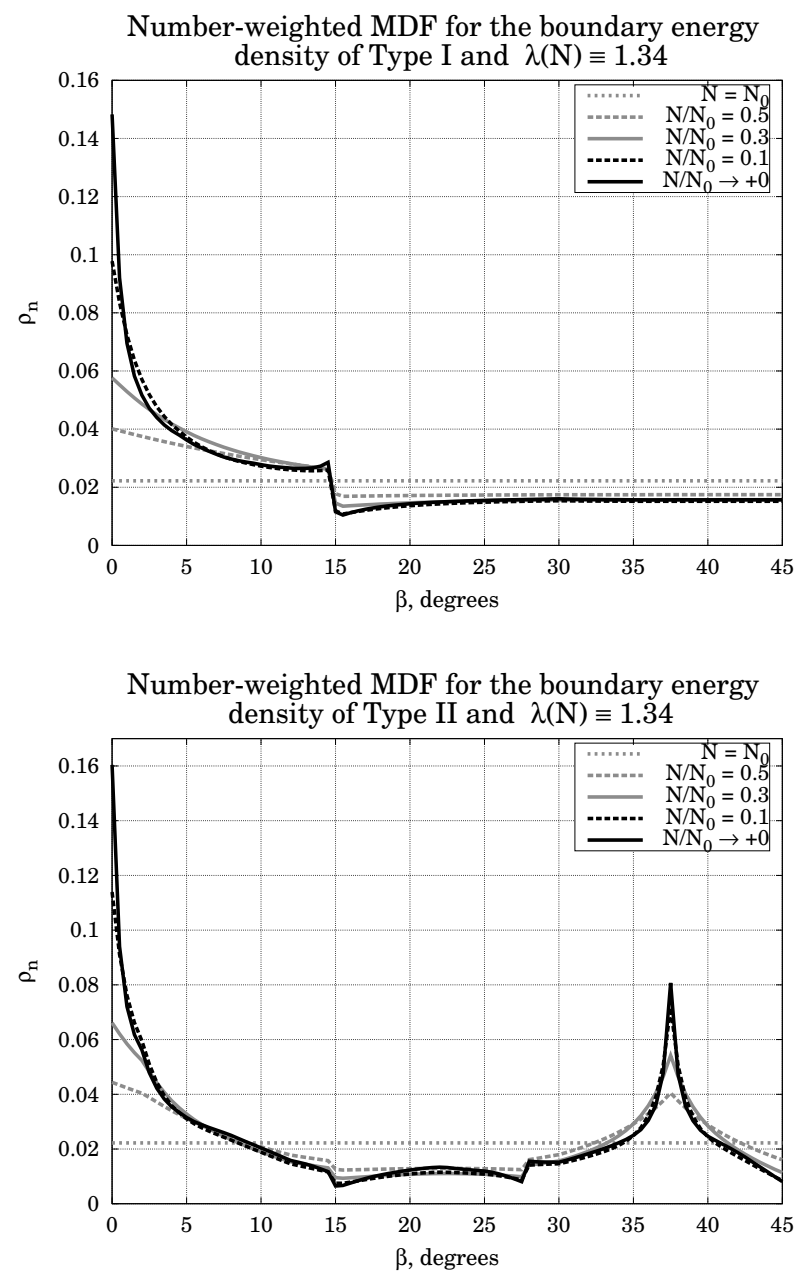

Number-weighted MDF for the boundary energy density of Type III and $\lambda(\mathrm{N}) \equiv 1.34$

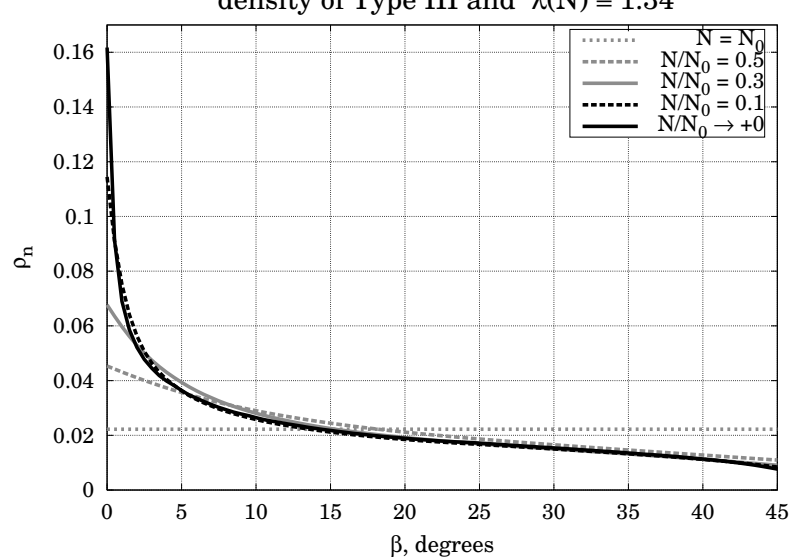

Figure 6: Number-weighted MDF in case $\mathbf{A}$. 
Number-weighted MDF for the boundary energy density of Type I and $\lambda(\mathrm{N})$ determined by (30) with

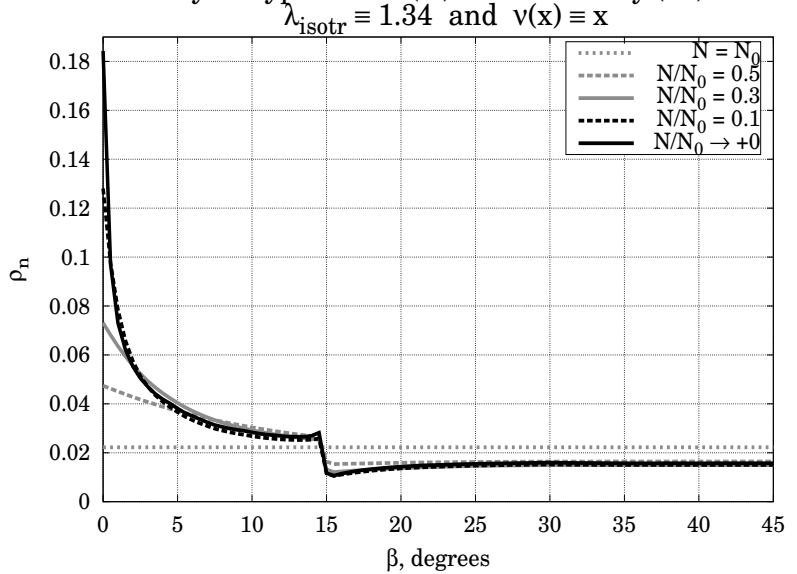

Number-weighted MDF for the boundary energy density of Type II and $\lambda(\mathrm{N})$ determined by (30) with

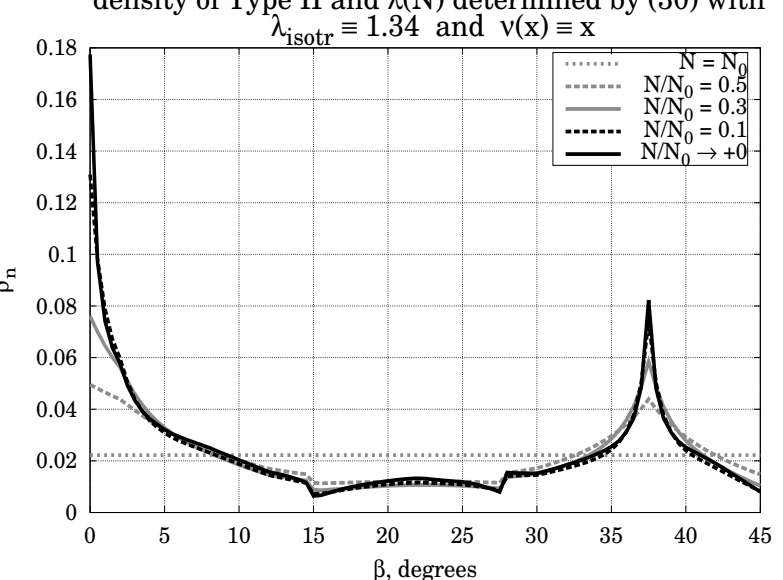

Number-weighted MDF for the boundary energy density of Type III and $\lambda(N)$ determined by (30) with

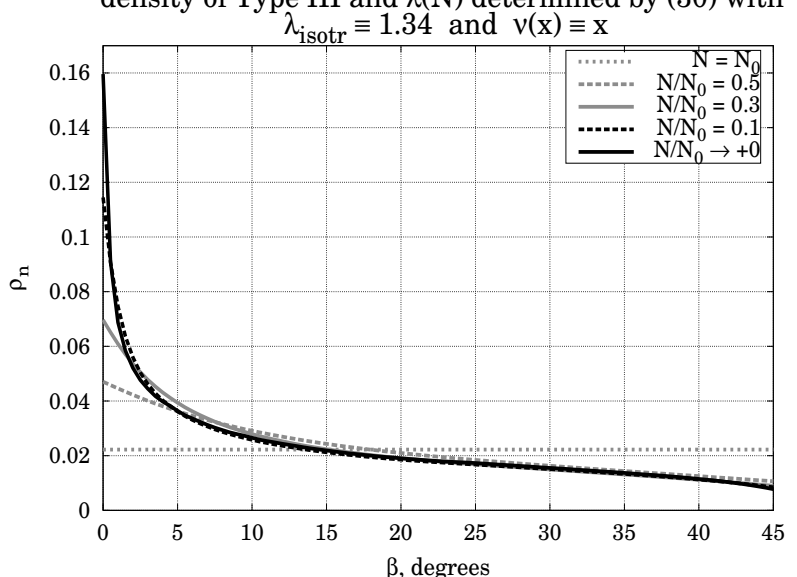

Figure 7: Number-weighted MDF in case $\mathbf{B}$. 

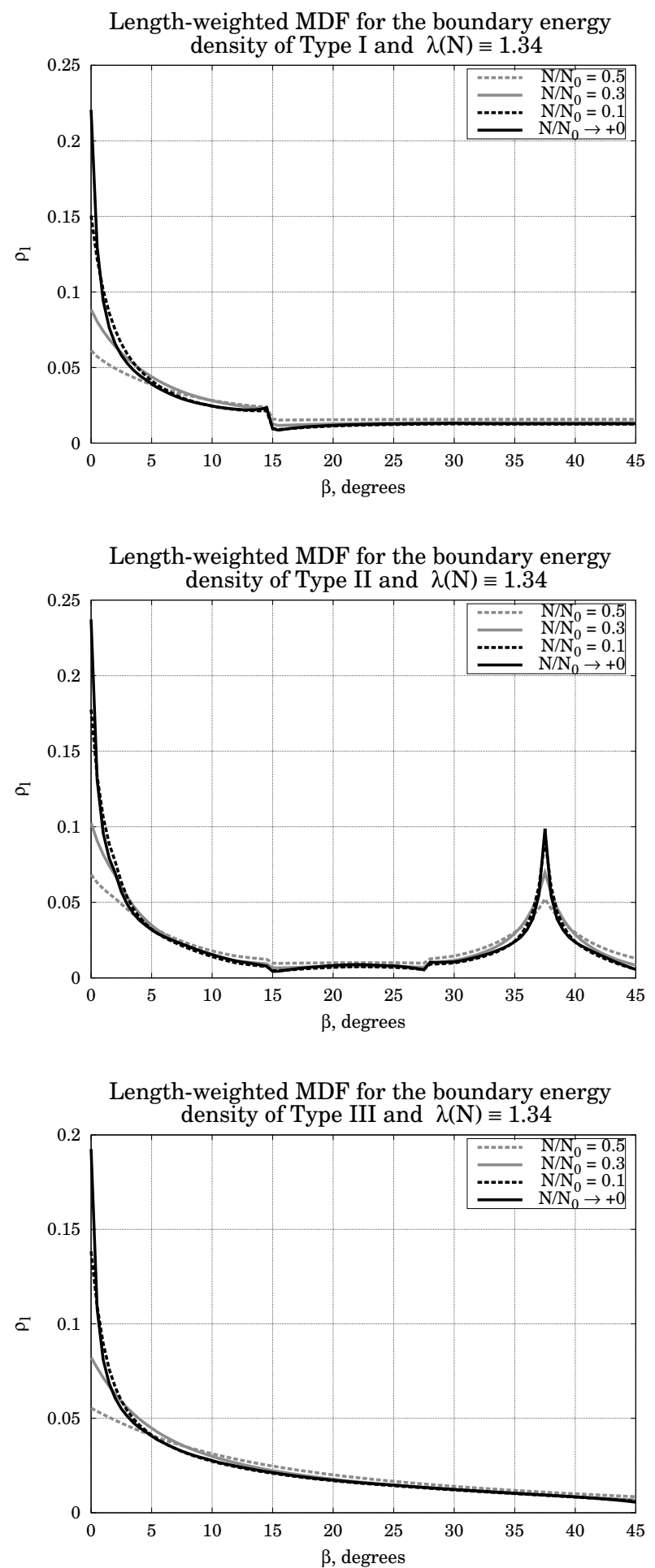

Figure 8: Length-weighted MDF in case $\mathbf{A}$ 
Length-weighted MDF for the boundary energy density of Type I and $\lambda(\mathrm{N})$ determined by (30) with

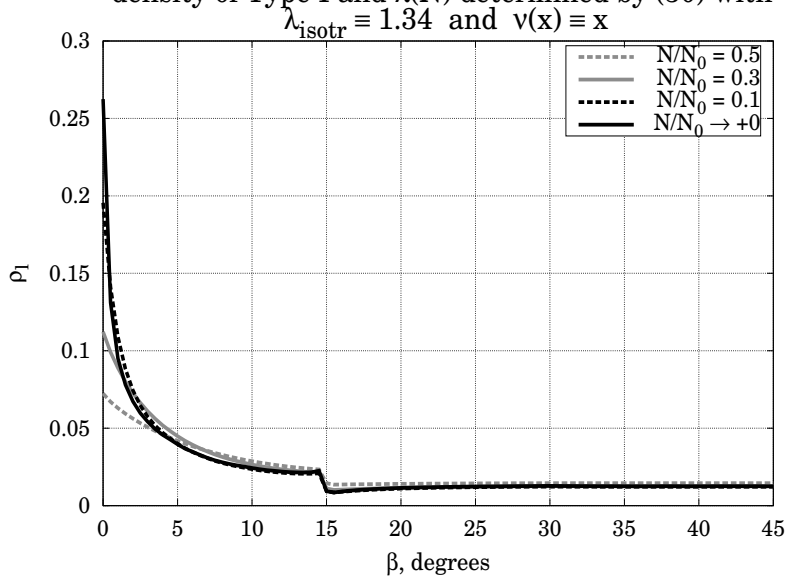

Length-weighted MDF for the boundary energy density of Type II and $\lambda(\mathrm{N})$ determined by (30) with

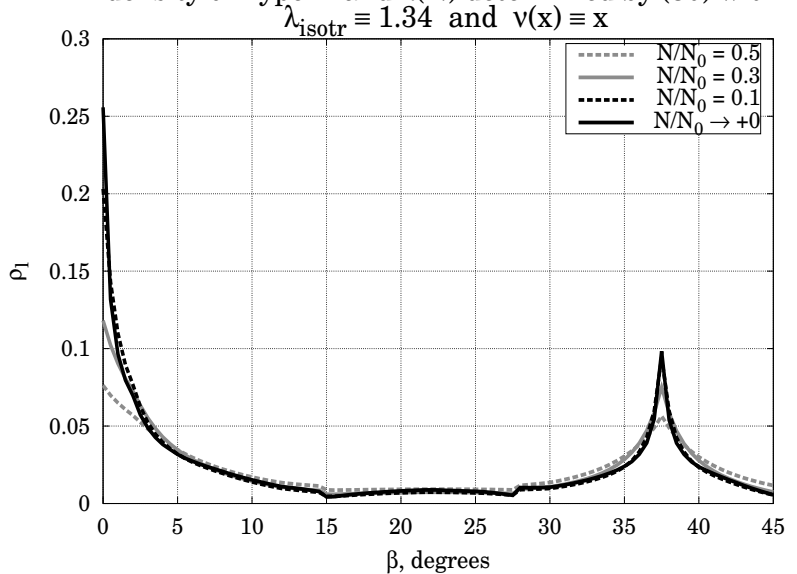

Length-weighted MDF for the boundary energy density of Type III and $\lambda(\mathrm{N})$ determined by (30) with

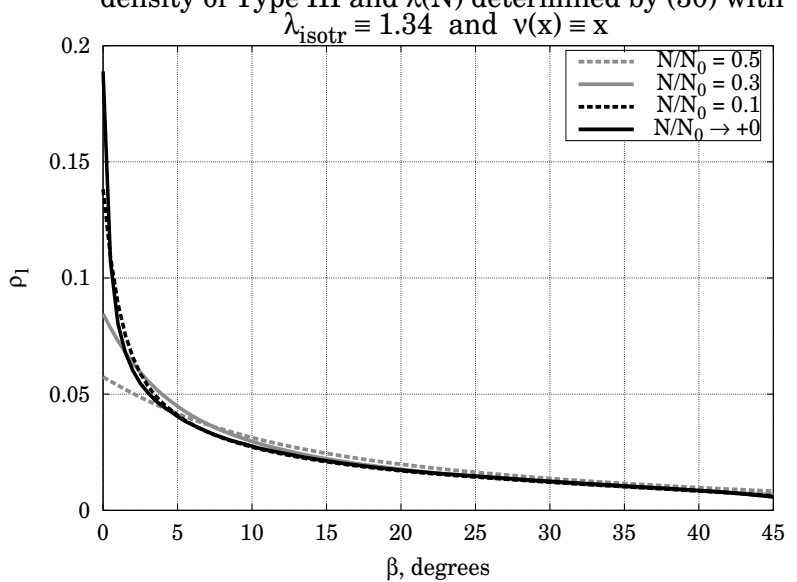

Figure 9: Length-weighted $M D F$ in case $\mathbf{B}$ 

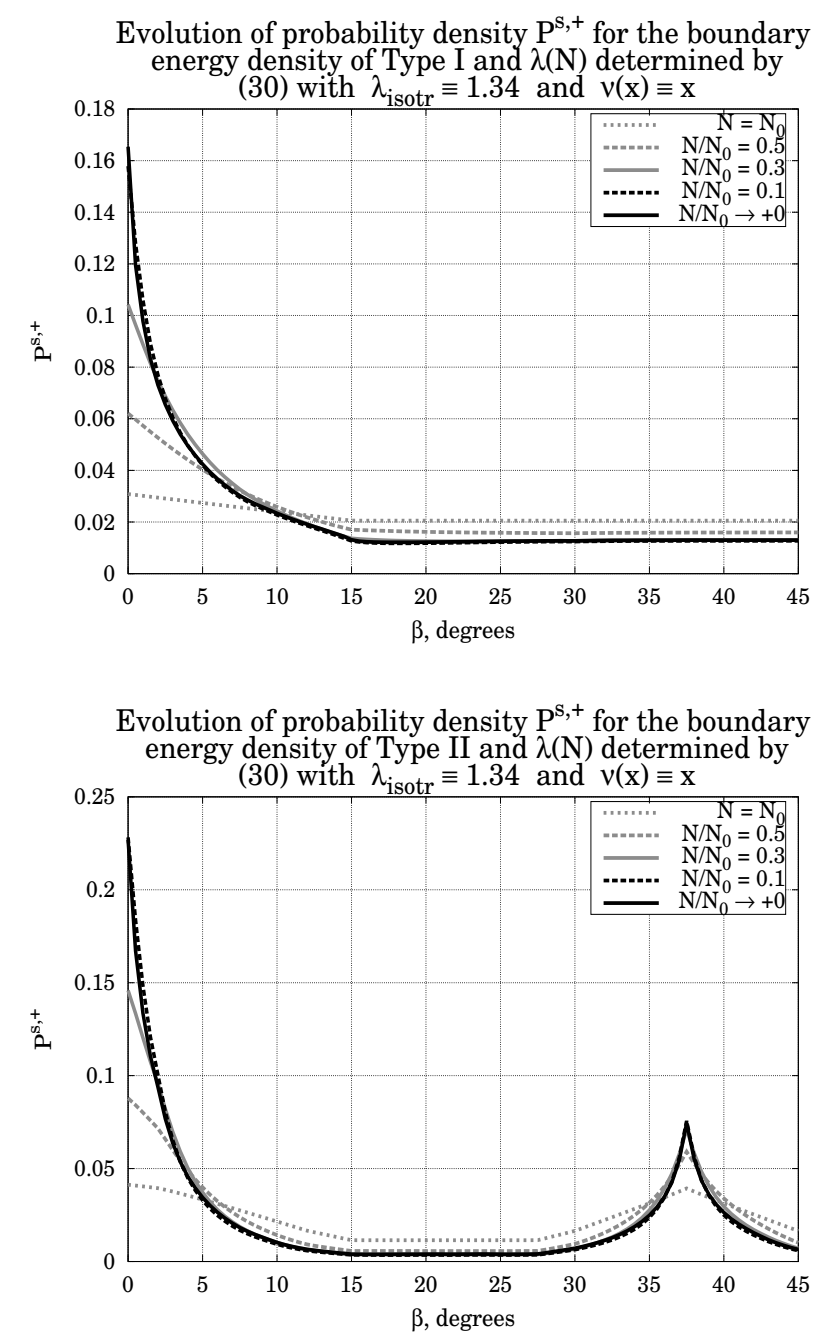

Evolution of probability density $\mathrm{P}^{\mathrm{s},+}$ for the boundary energy density of Type III and $\lambda(\mathrm{N})$ determined by

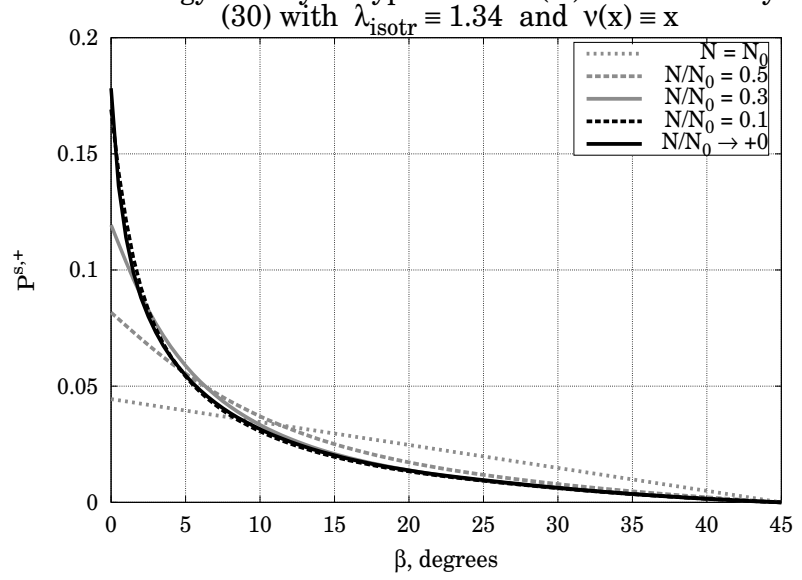

Figure 10: Evolution of probability density $P^{s,+}(\cdot, N)$ in case $\mathbf{B}$. 
Evolution of probability density $\mathrm{P}^{\mathrm{S},-}$ for the boundary energy density of Type I and $\lambda(\mathrm{N})$ determined by

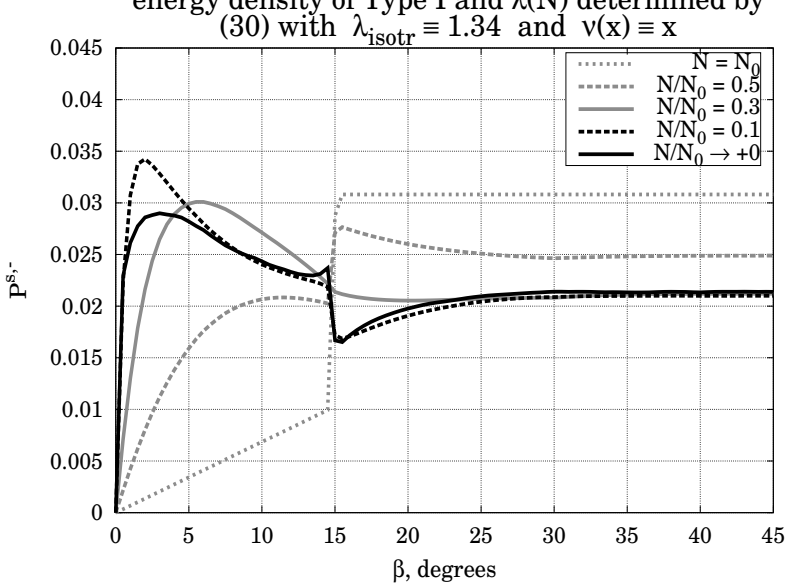

Evolution of probability density $\mathrm{P}^{\mathrm{s},-}$ for the boundary energy density of Type II and $\lambda(\mathrm{N})$ determined by

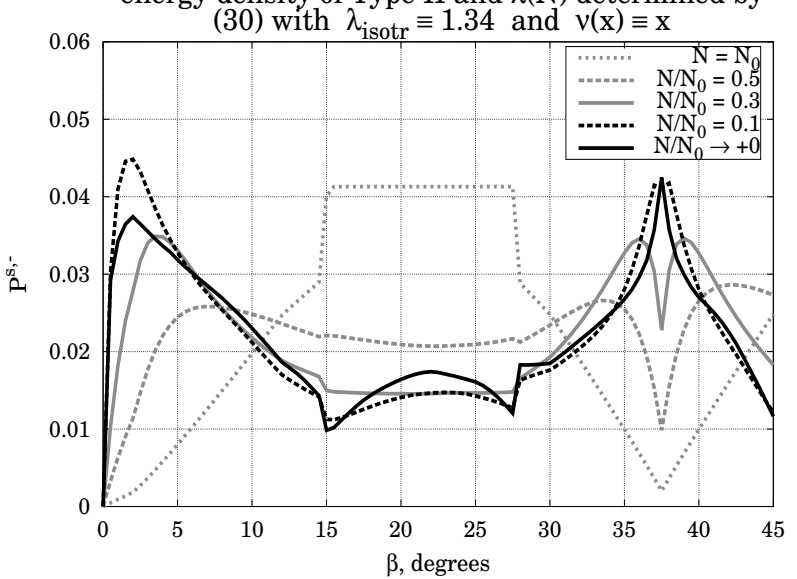

Evolution of probability density $\mathrm{P}^{\mathrm{s},-}$ for the boundary energy density of Type III and $\lambda(\mathrm{N})$ determined by

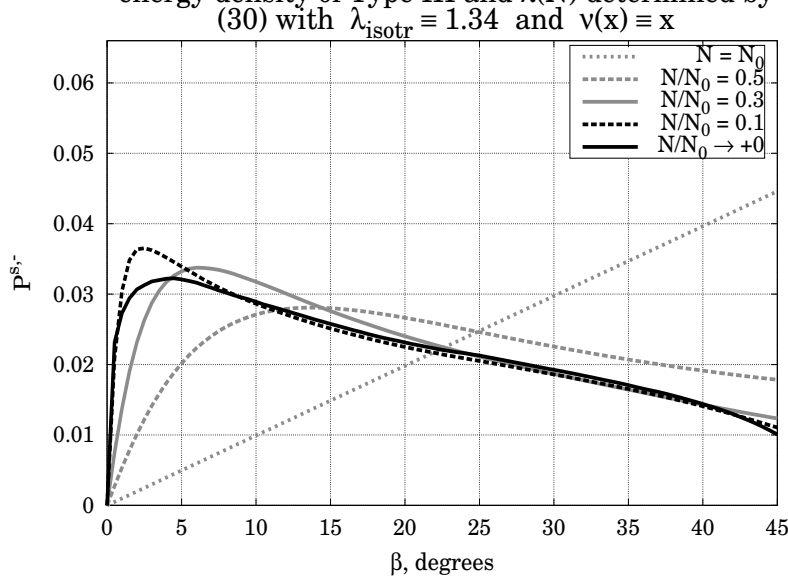

Figure 11: Evolution of probability density $P^{s,-}(\cdot, N)$ in case $\mathbf{B}$ 
Evolution of probability density $\mathrm{P}_{1}^{\mathrm{d}}$ for the boundary energy density of Type I and $\lambda(\mathrm{N})$ determined by

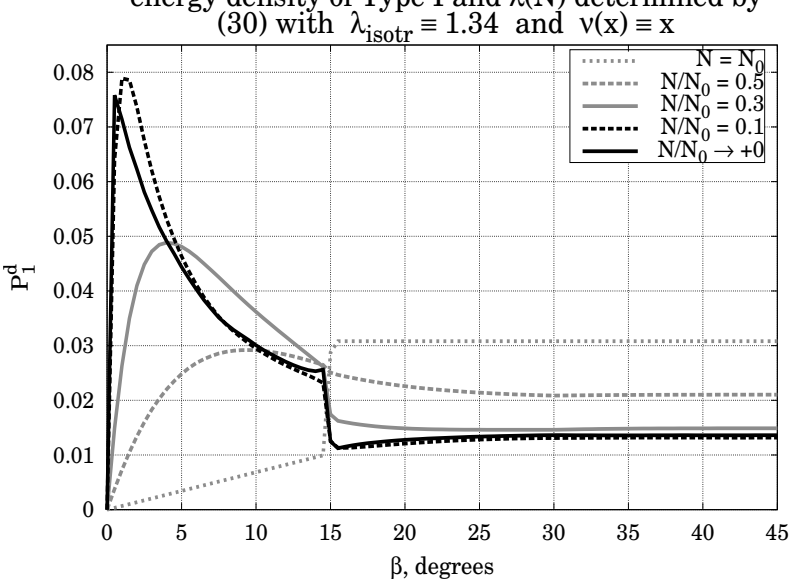

Evolution of probability density $\mathrm{P}_{1}^{\mathrm{d}}$ for the boundary energy density of Type II and $\lambda(\mathrm{N})$ determined by

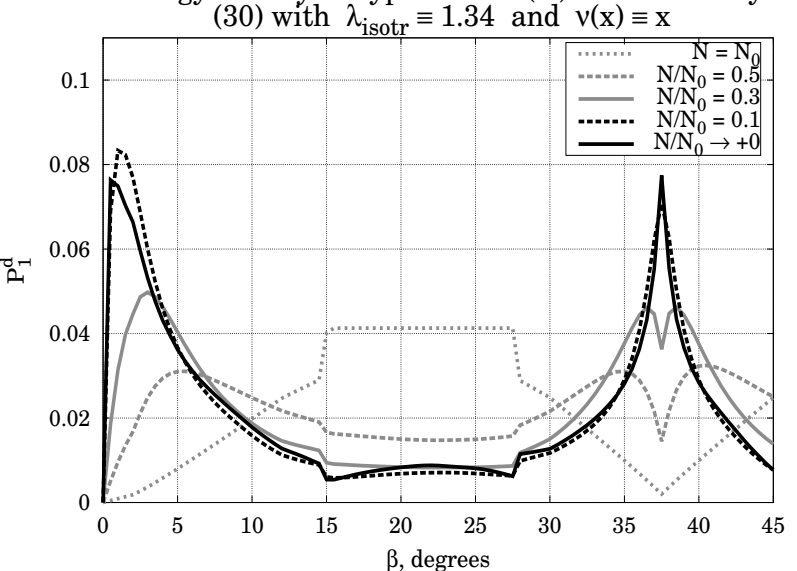

Evolution of probability density $\mathrm{P}_{1}^{\mathrm{d}}$ for the boundary energy density of Type III and $\lambda(\mathrm{N})$ determined by

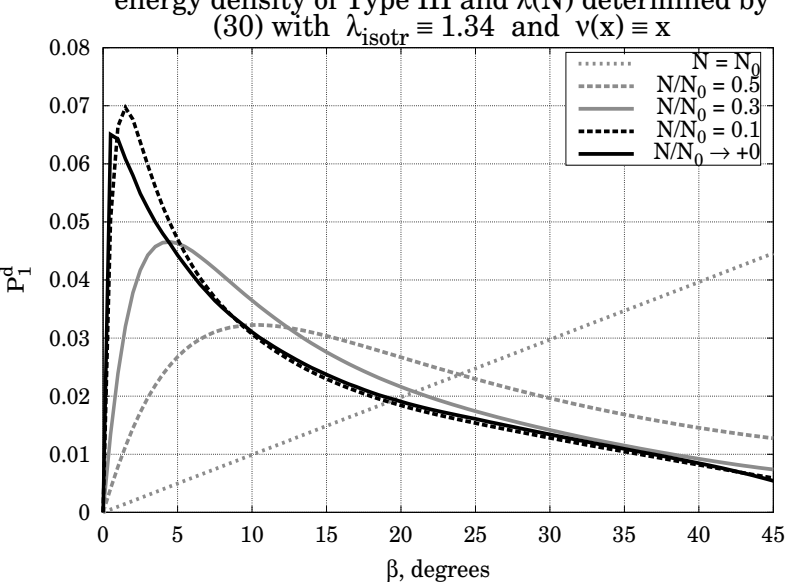

Figure 12: Evolution of probability density $P_{1}^{d}(\cdot, N)$ in case $\mathbf{B}$. 
Evolution of probability density $\mathrm{P}_{2}^{\mathrm{d}}$ for the boundary energy density of Type I and $\lambda(\mathrm{N})$ determined by

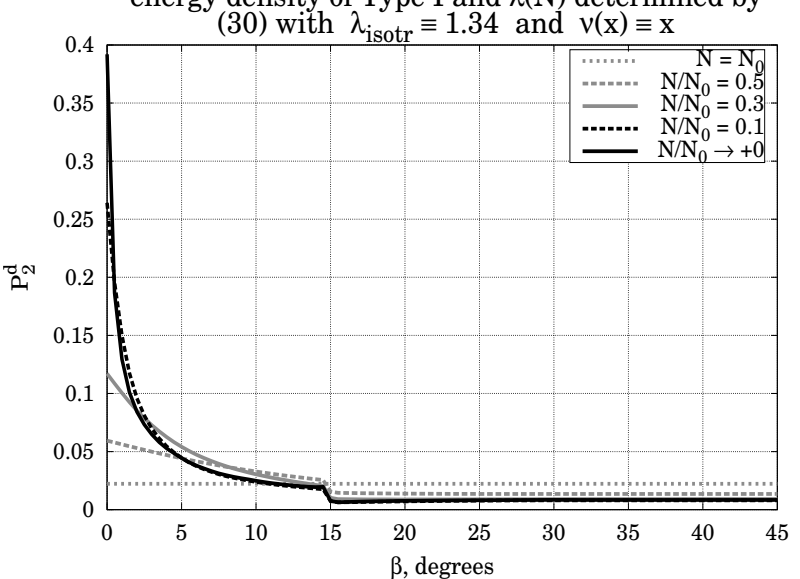

Evolution of probability density $\mathrm{P}_{2}^{\mathrm{d}}$ for the boundary energy density of Type II and $\lambda(\mathrm{N})$ determined by

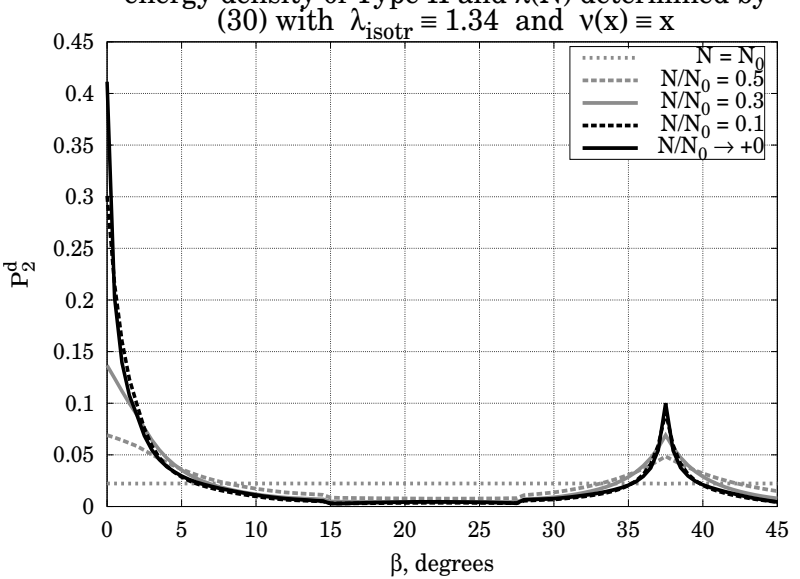

Evolution of probability density $\mathrm{P}_{2}^{\mathrm{d}}$ for the boundary energy density of Type III and $\lambda(\mathrm{N})$ determined by

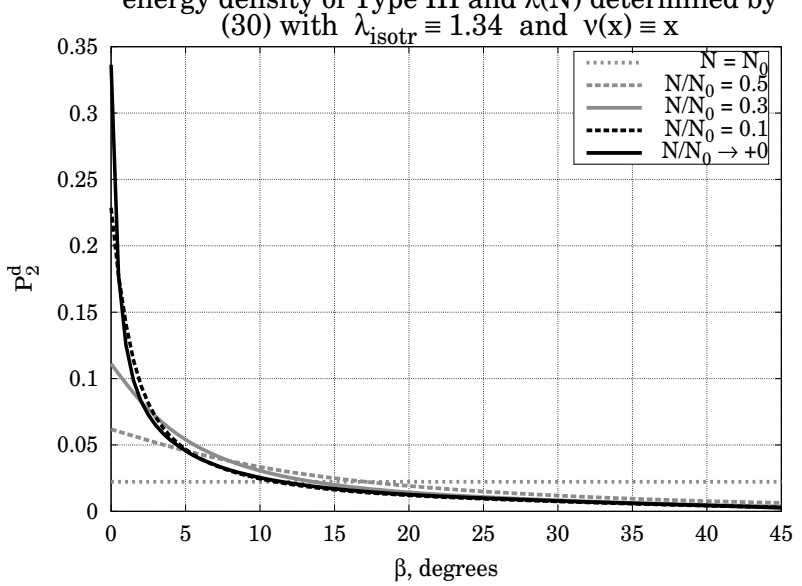

Figure 13: Evolution of probability density $P_{2}^{d}(\cdot, N)$ in case $\mathbf{B}$. 

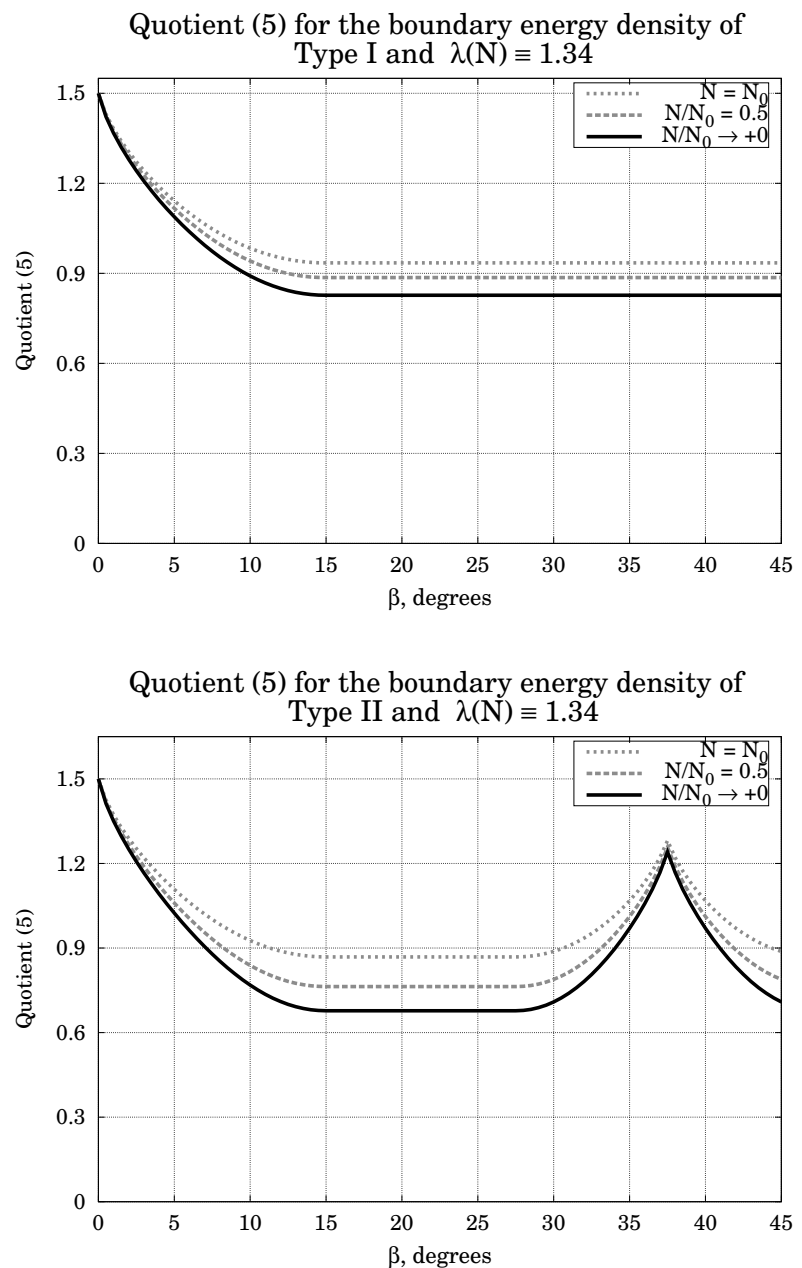

Quotient (5) for the boundary energy density of

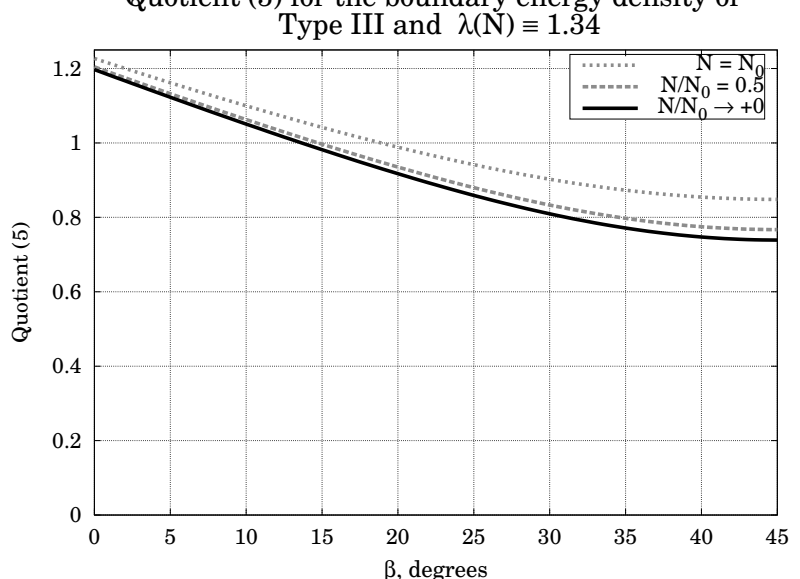

Figure 14: Estimate (33) of quotient (5) in case $\mathbf{A}$. 
Quotient (5) for the boundary energy density of

Type I and $\lambda(\mathrm{N})$ determined by (30) with

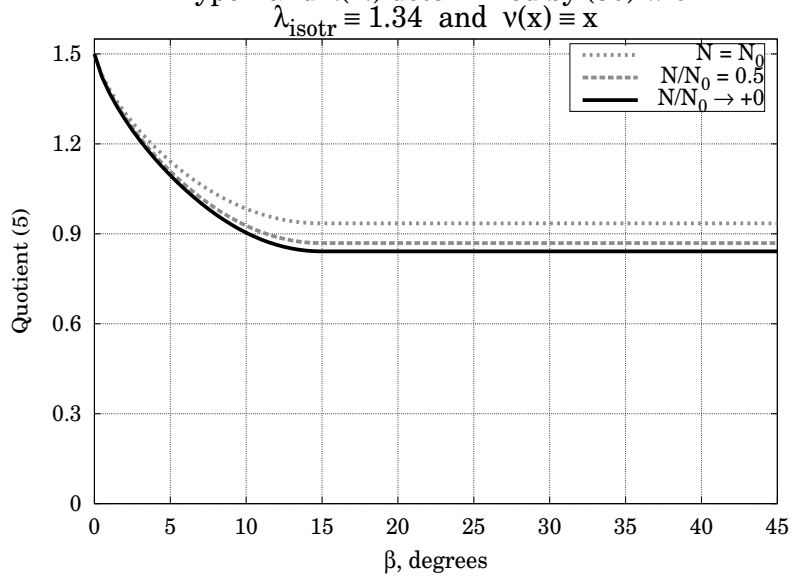

Quotient (5) for the boundary energy density of Type II and $\lambda(\mathrm{N})$ determined by $(30)$ with

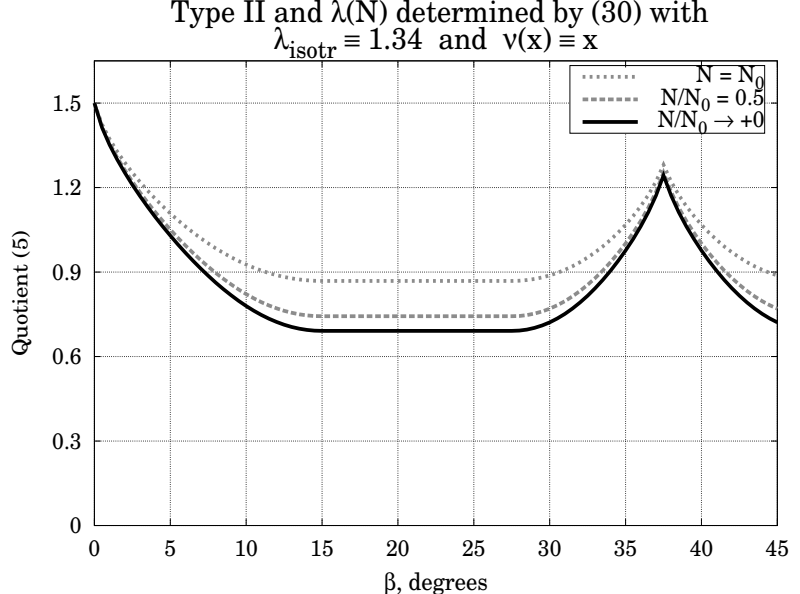

Quotient (5) for the boundary energy density of Type III and $\lambda(\mathrm{N})$ determined by (30) with

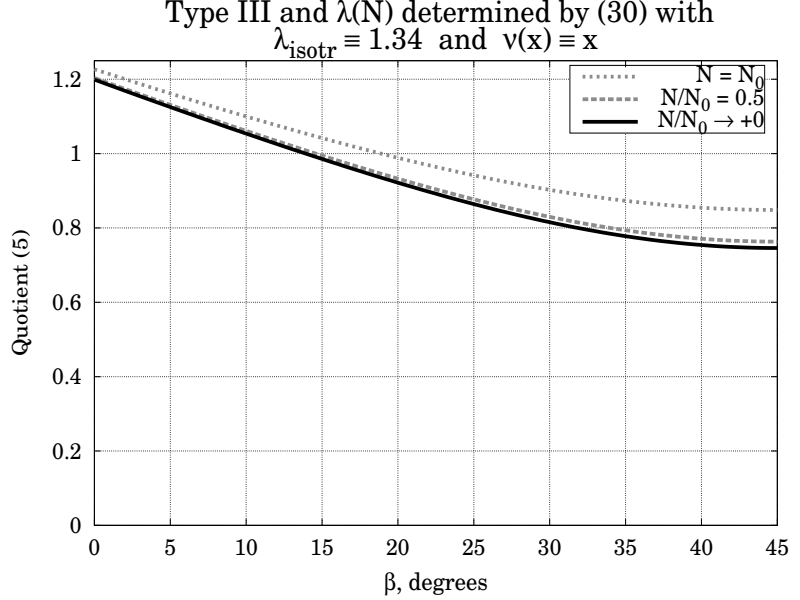

Figure 15: Estimate (33) of quotient (5) in case $\mathbf{B}$. 
Evolution of low-energy and high-energy boundaries' fractions in case of the boundary energy density of

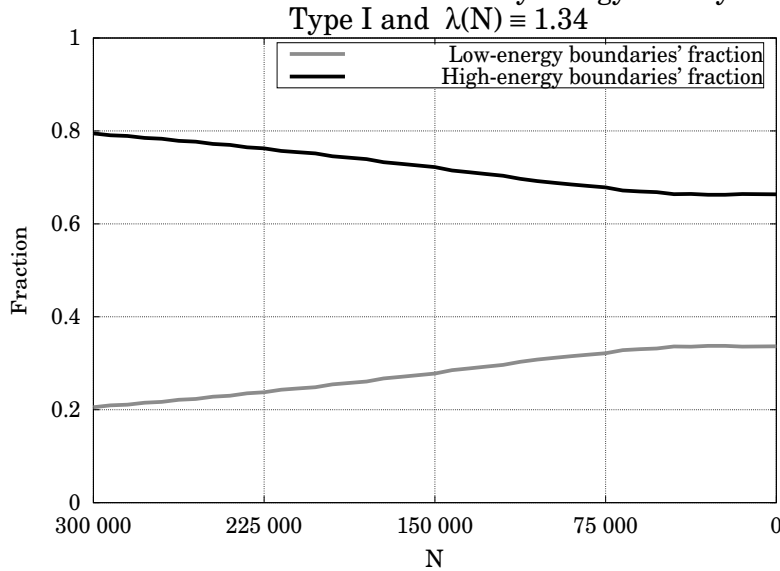

Evolution of low-energy and high-energy boundaries' fractions in case of the boundary energy density of

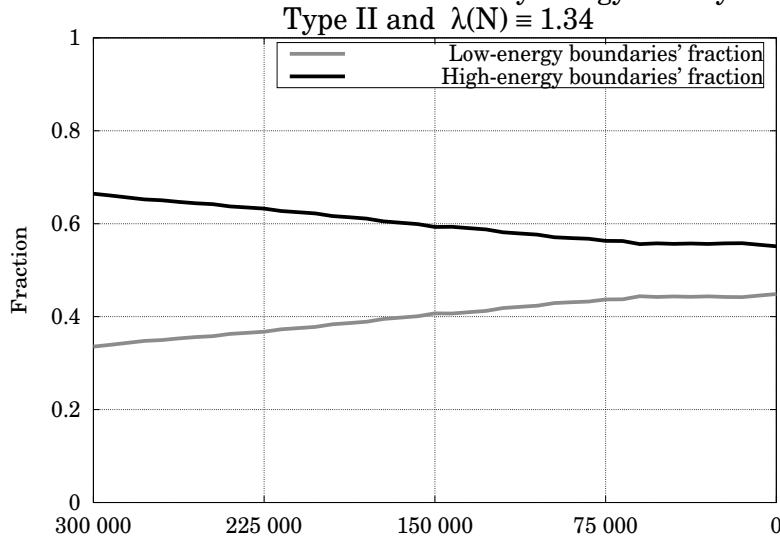

$\mathrm{N}$

Evolution of low-energy and high-energy boundaries' fractions in case of the boundary energy density of

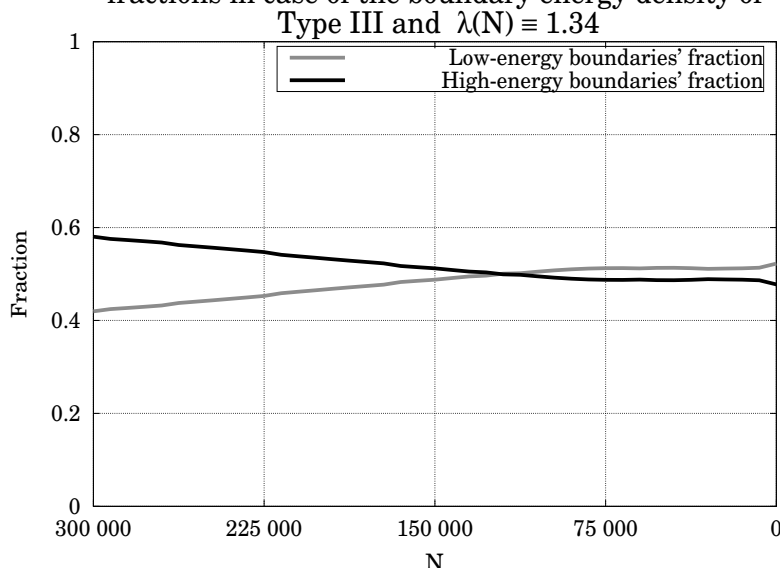

Figure 16: Evolution of low-energy and high-energy boundaries' fractions $\kappa_{\mathrm{le}}(N), \kappa_{\mathrm{he}}(N)$ in case $\mathbf{A}$. 
Evolution of low-energy and high-energy boundaries' fractions in case of the boundary energy density of

Type I and $\lambda(\mathrm{N})$ determined by (30) with $\lambda_{\text {isotr }}=1.34$ and $v(\mathrm{x}) \equiv \mathrm{x}$

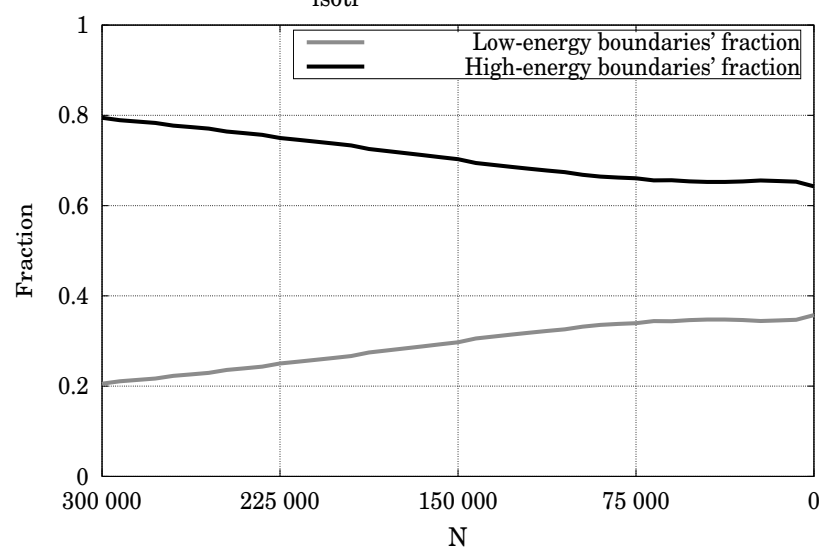

Evolution of low-energy and high-energy boundaries' fractions in case of the boundary energy density of Type II and $\lambda(\mathrm{N})$ determined by (30) with $\lambda_{\text {isotr }}=1.34$ and $v(x) \equiv \mathrm{x}$

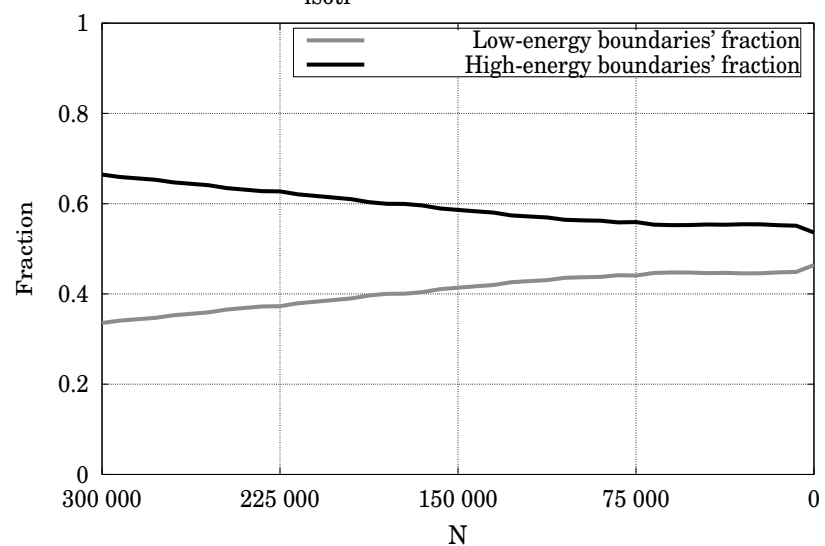

Evolution of low-energy and high-energy boundaries' fractions in case of the boundary energy density of Type III and $\lambda(\mathrm{N})$ determined by (30) with $\lambda_{\text {isotr }}=1.34$ and $v(\mathrm{x}) \equiv \mathrm{x}$

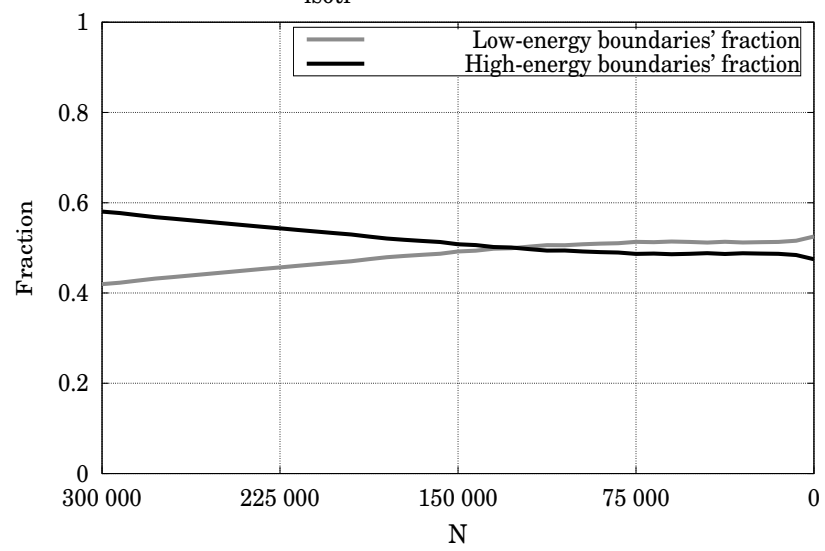

Figure 17: Evolution of low-energy and high-energy boundaries' fractions $\kappa_{\mathrm{le}}(N), \kappa_{\mathrm{he}}(N)$ in case $\mathbf{B}$. 
Evolution of the energy density averaged over all existing boundaries in the system (Type I of the energy

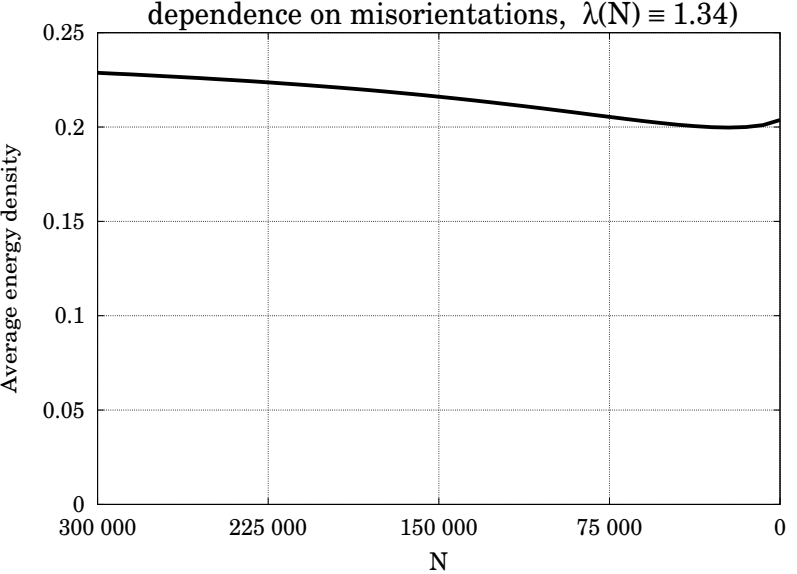

Evolution of the energy density averaged over all existing boundaries in the system (Type II of the energy

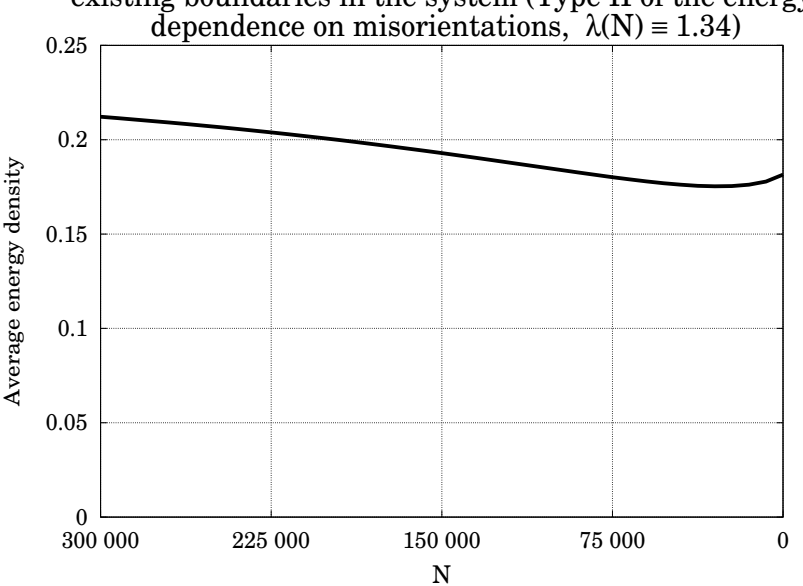

Evolution of the energy density averaged over all existing boundaries in the system (Type III of the energy

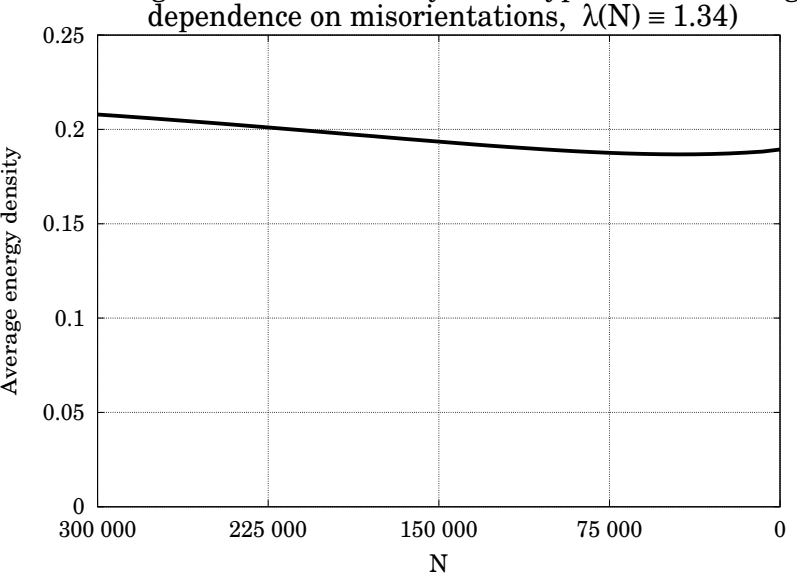

Figure 18: Evolution of the average energy density $\bar{\gamma}(N)$ in case $\mathbf{A}$. 
Evolution of the energy density averaged over all existing boundaries in the system (Type I of the energy dependence on misorientations, $\lambda(\mathrm{N})$ determined by (30) with $\lambda_{\text {isotr }}=1.34$ and $\left.v(x) \equiv x\right)$

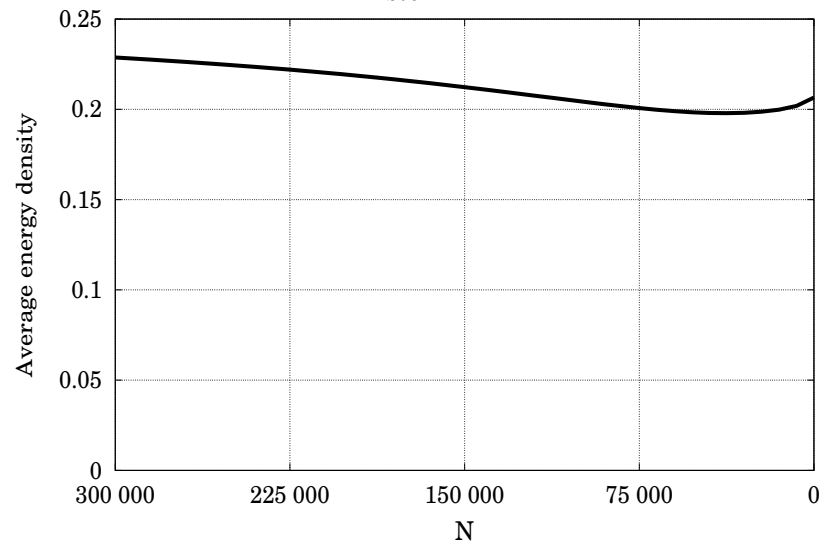

Evolution of the energy density averaged over all existing boundaries in the system (Type II of the energy dependence on misorientations, $\lambda(\mathrm{N})$ determined by (30) with $\lambda_{\text {isotr }}=1.34$ and $\left.v(x) \equiv x\right)$

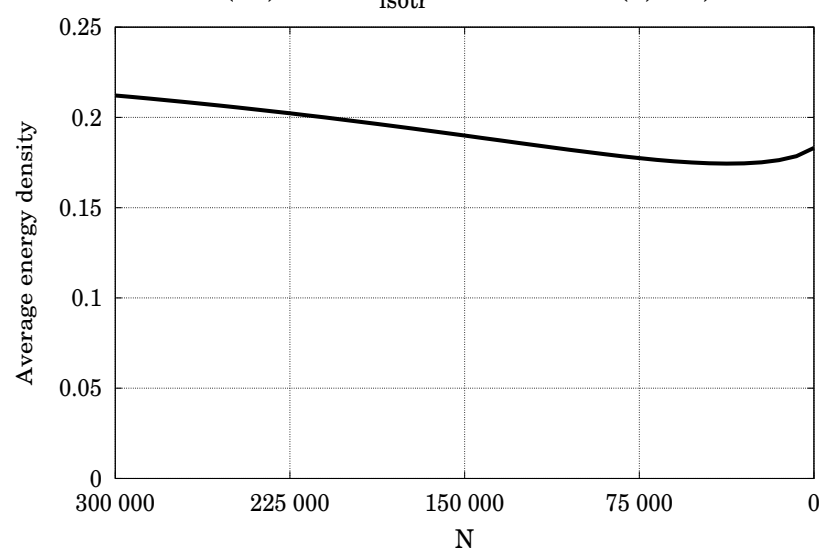

Evolution of the energy density averaged over all existing boundaries in the system (Type III of the energy dependence on misorientations, $\lambda(\mathrm{N})$ determined by (30) with $\lambda_{\text {isotr }}=1.34$ and $\left.v(x) \equiv x\right)$

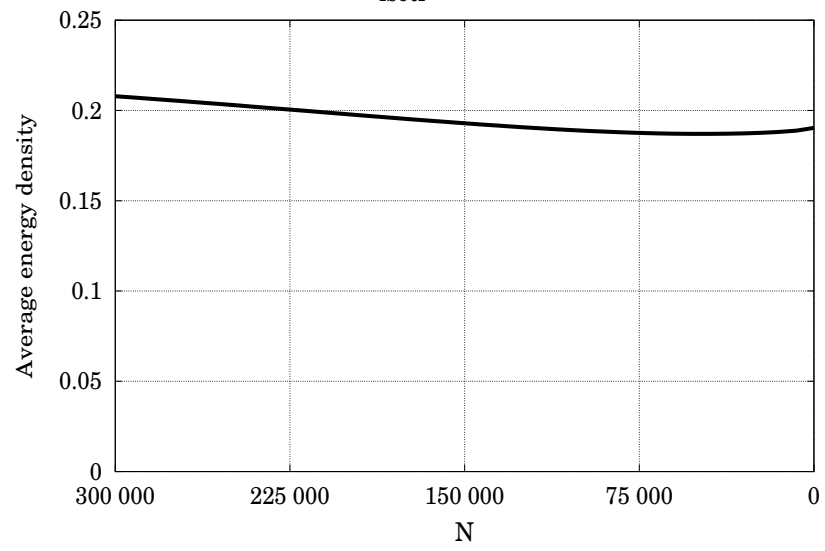

Figure 19: Evolution of the average energy density $\bar{\gamma}(N)$ in case $\mathbf{B}$. 
Evolution of the ratio of the neighbor switchings' rate to the grain disappearances' rate for the boundary energy density of Type I according to (30) with $\lambda_{\text {isotr }}=1.34$ and $\mathrm{v}(\mathrm{x}) \equiv \mathrm{x}$

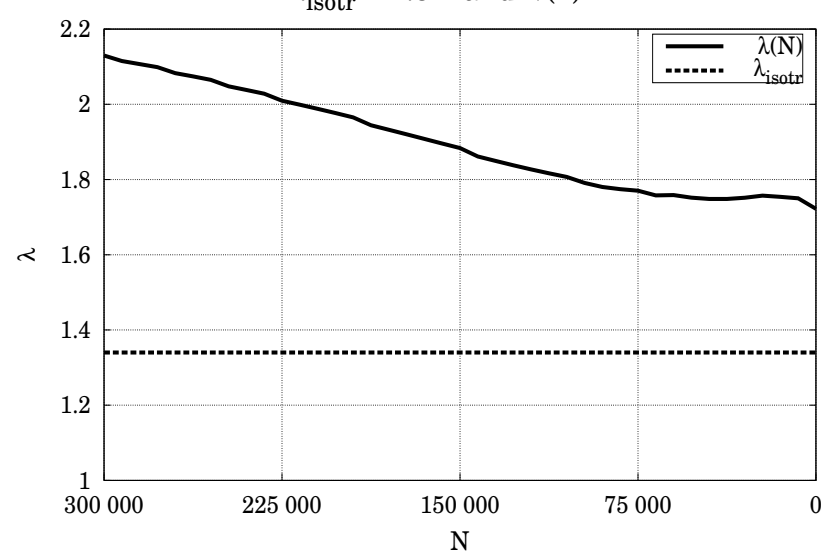

Evolution of the ratio of the neighbor switchings' rate to the grain disappearances' rate for the boundary energy density of Type II according to (30) with $\lambda_{\text {isotr }}=1.34$ and $v(x) \equiv x$

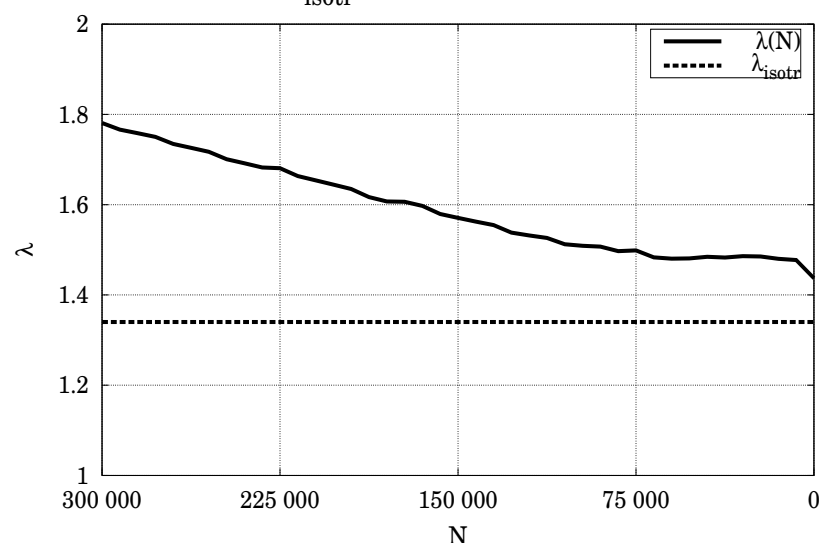

$\mathrm{N}$

Evolution of the ratio of the neighbor switchings' rate to the grain disappearances' rate for the boundary energy density of Type III according to (30) with $\lambda_{\text {isotr }}=1.34$ and $\mathrm{v}(\mathrm{x}) \equiv \mathrm{x}$

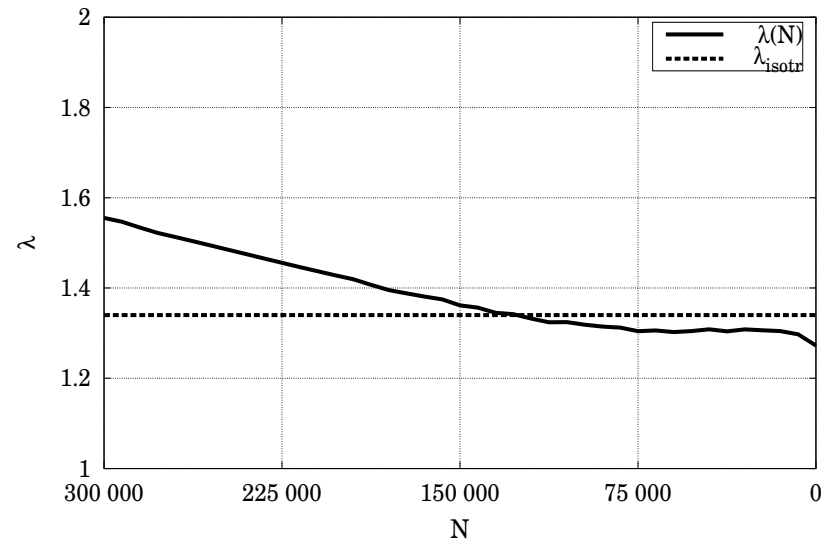

Figure 20: Evolution of the ratio $\lambda(N)$ of the neighbor switchings' rate to the grain disappearances' rate in case $\mathbf{B}$. 